\title{
Reconstruction of the Genesis Entry
}

\author{
Prasun N. Desai ${ }^{(1)}$, Garry D. Qualls ${ }^{(2)}$, Mark Schoenenberger ${ }^{(3)}$ \\ ${ }^{(1)}$ NASA Langley Research Center, MS 489, Hampton, VA 23681-2199, USA \\ Email: prasun.n.desai@nasa.gov \\ ${ }^{(2)}$ NASA Langley Research Center, MS 458, Hampton, VA 23681-2199, USA \\ Email: garry.d.qualls@nasa.gov \\ ${ }^{(3)}$ NASA Langley Research Center, MS 489, Hampton, VA 23681-2199, USA \\ Email: mark.schoenenberger-1@nasa.gov
}

\begin{abstract}
An overview of the reconstruction analyses performed for the Genesis capsule entry is described. The results indicate that the actual entry prior to the drogue deployment failure was very close to the pre-entry predictions. The capsule landed $8.3 \mathrm{~km}$ south of the desired target at Utah Test and Training Range. Analysis on infrared video footage (obtained from the tracking stations) during the descent estimated the onset of the capsule tumble at Mach 0.9. Frequency analysis on the infrared video data indicates that the aerodynamics generated for the Genesis capsule reasonably predicted the drag and static stability. Observations of the heatshield support the pre-entry simulation estimates of a small hypersonic angles-ofattack, since there is very little, if any, charring of the shoulder region or the aftbody. Through this investigation, an overall assertion can be made that all the data gathered from the Genesis entry is consistent with flight performance close to the nominal pre-entry prediction. Consequently, the design principles and methodologies utilized for the flight dynamics, aerodynamics, and aerothermodynamics analyses have been corroborated.
\end{abstract}




\subsection{Introduction}

Genesis, the fifth of NASA's Discovery class missions, was launched on August 8, 2001. It is the first mission to return samples from beyond the Earth-moon system. Genesis was inserted into a halo orbit about the sun-Earth libration point (L1) where it collected solar wind particles over a period of approximately 29 months. Upon Earth return, the Genesis entry capsule containing the solar wind samples entered the Earth's atmosphere on the morning of September 8, 2004 at 15:52:47 UTC. Reference [1] provides an overview of the Earth return trajectory strategy.

Maneuver and targeting procedures prior to entry interface were nominal and placed the capsule on the expected flight path required for a successful entry profile for a mid-air recovery using a helicopter over the U.S. Air Force's Utah Test and Training Range (UTTR) in Northwest Utah [2, 3]. Figure 1 illustrates the nominal entry sequence.

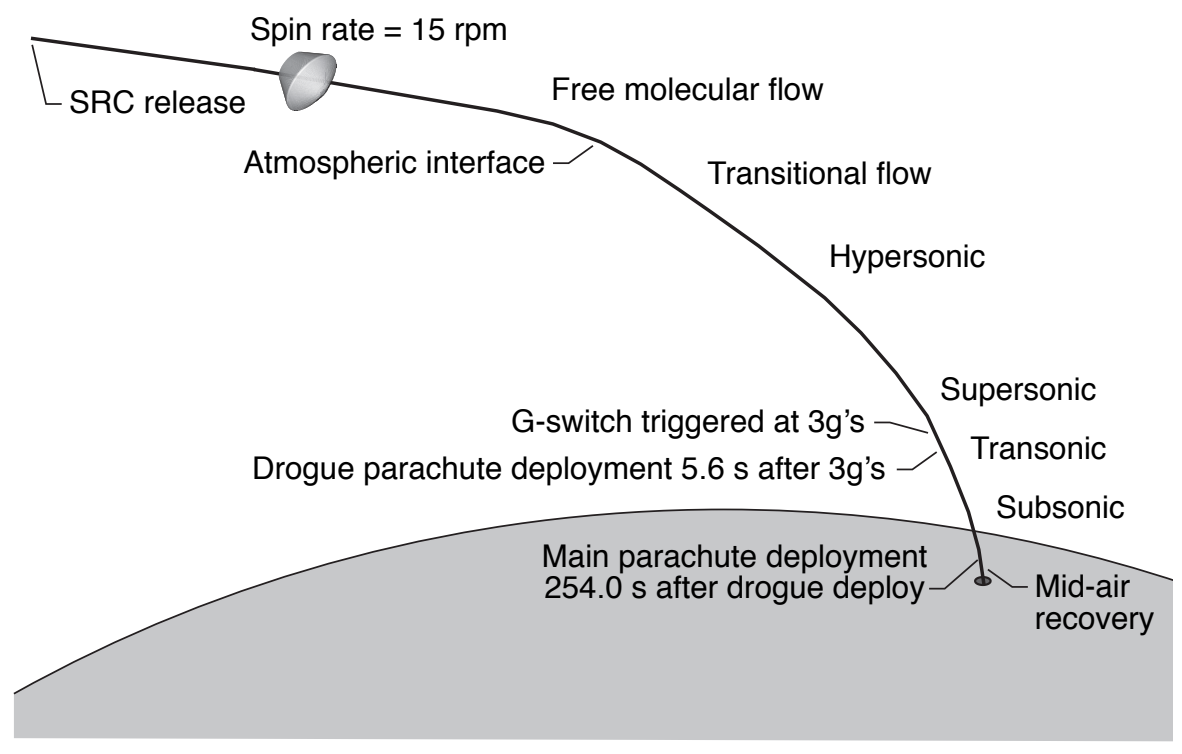

Figure 1. Nominal Genesis capsule entry sequence

Four hours prior to entry, the $205.6 \mathrm{~kg}$ Genesis capsule was spun-up to $15 \mathrm{rpm}$ and separated from the main spacecraft. The capsule has no active guidance or control system, so the spin-up is required to maintain its entry attitude (nominal 0 deg angle-of-attack) during coast. Throughout the atmospheric entry, the passive capsule relies solely on aerodynamic stability for performing a controlled descent through all aerodynamic flight regimes: free molecular, hypersonic-transitional, hypersonic-continuum, supersonic, transonic, and subsonic [4]. Therefore, the capsule must possess sufficient aerodynamic stability to minimize any angle-of-attack excursions during the severe heating environment. Additionally, this stability must persist through the transonic and subsonic regimes to maintain a controlled attitude at drogue and main parachute deployment. The inertial entry velocity and flight-path angle for Genesis were 11.04 $\mathrm{km} / \mathrm{sec}$ and $-8.0 \mathrm{deg}$, respectively.

Unfortunately, due to a hardware malfunction during the descent, the signal to initiate drogue parachute deployment failed and the capsule subsequently tumbled and impacted the surface. Following the failure, a reconstruction effort was initiated in an effort to assess how well the flight dynamics, aerodynamics, and aerothermodynamics predictions (performed during the development phase) compared to the actual entry. 
This paper provides an overview of the findings from a reconstruction analysis of the Genesis capsule entry. First, a comparison of the atmospheric properties (density and winds) encountered during the entry to the pre-entry profile is presented. The analysis that was performed on the video footage (obtained from the tracking stations at UTTR) during the descent is then described from which the Mach number at the onset of the capsule tumble was estimated following the failure of the drogue parachute deployment. Next, an assessment of the Genesis capsule aerodynamics that was extracted from the video footage is discussed, followed by a description of the capsule hypersonic attitude that must have occurred during the entry based on examination of the recovered capsule heatshield. Lastly, the entry trajectory reconstruction that was performed is presented.

\subsection{Final Landing Location}

The impact point of the Genesis capsule was $8.3 \mathrm{~km}$ south of the desired target as seen in Fig. 2. Also, shown in Fig. 2 is the pre-entry predicted nominal landing location, as well as the final $99 \%$ landing ellipse calculated during final approach [3]. The overall 99\% landing ellipse was calculated to be $41.9 \mathrm{~km}$ by $21.1 \mathrm{~km}$ having an azimuth orientation angle of $137.2 \mathrm{deg}$ (measured clockwise positive from North). The $8.3 \mathrm{~km}$ downrange distance of the final impact point from the target was within approximately 1- $\sigma$ of the calculated landing ellipse.

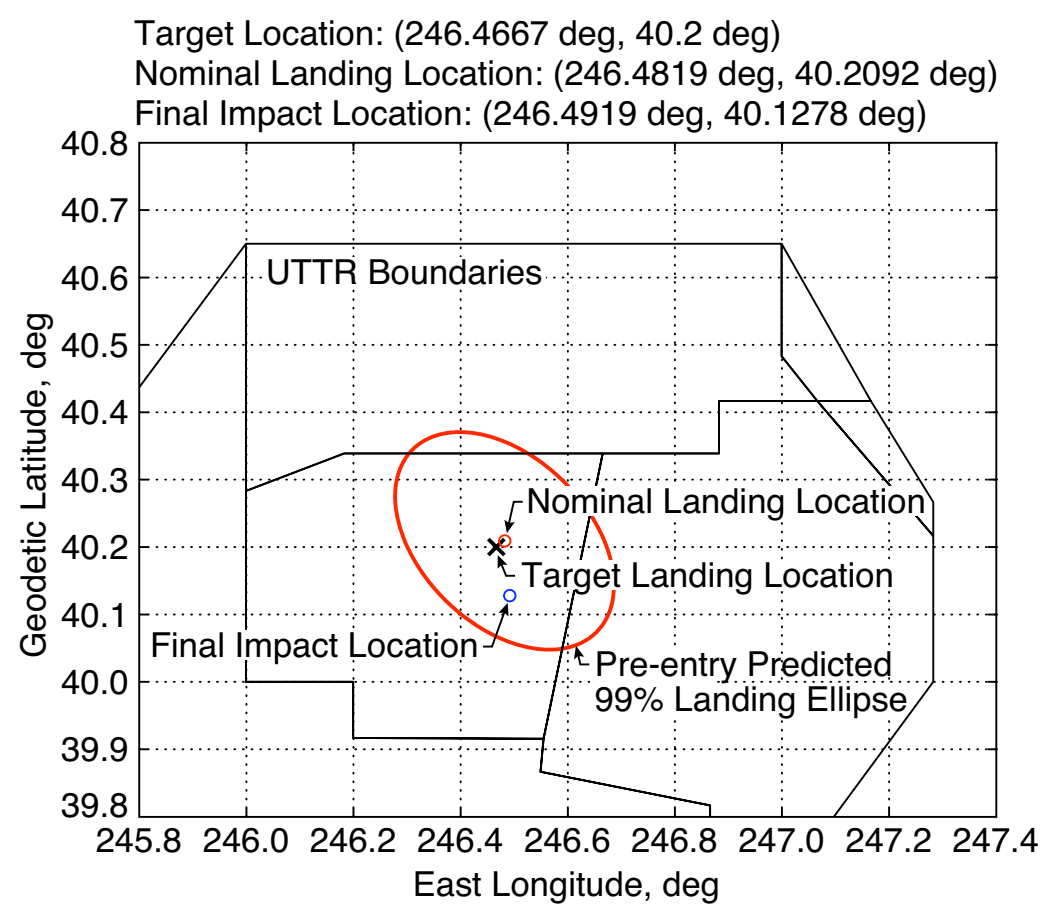

Figure 2. Final Genesis capsule landing location

Although, the Genesis capsule landed very close to the pre-entry predicted landing location, an understanding of the hypersonic flight is of great interest. Specifically, assessing the aerodynamics, flight dynamics, and aerothermodynamic performance of the capsule is desired to gain confidence in the design principles and methodologies that are utilized for the design and development phase of entry vehicles. The subsequent section provides an overview of each discipline. 


\subsection{Atmosphere Comparison}

The Earth atmosphere model utilized by Genesis for the entry trajectory design and analysis was the Global Reference Atmospheric Model - 1995 Version (GRAM-95) [5]. This model is an amalgam of three empirically based global atmospheric data sets of the Earth that can produce an atmosphere profile of density, temperature, pressure, and winds (northward, eastward, and vertical wind components) as a function of altitude for a given date, time, and positional location about the Earth. GRAM-95 produces a representative atmosphere taking into account variations in diurnal, seasonal, and positional information for a given trajectory to produce nominal density, temperature, pressure, and wind profiles along the trajectory flight track. GRAM-95 is not a predictive model, but rather provides a representative atmosphere profile for the given date, time, and positional inputs. A profile is generated based on historical data for a given date, time, and location. In addition, GRAM-95 also provides statistical perturbations for all the atmosphere parameters.

Four hours prior to the capsule entry, a balloon was launched from UTTR to obtain measurements of the atmospheric properties over the range. The balloon measured density data is plotted in Fig. 3 as a percentage of the nominal profile obtained from the GRAM-95 model. Note, measurements were only available for altitudes up to $34 \mathrm{~km}$. Also depicted in Fig. 3 are the upper and lower 3- $\sigma$ boundaries of the possible density variation (as a percentage of the nominal profile) produced by the GRAM-95 model for the Genesis entry date and time. Figure 4 is an expanded view of the altitude band of the measured data set. As seen, the measured density for altitudes below $34 \mathrm{~km}$ was very close to the nominal profile produced by the GRAM-95 model and falls well within the $3-\sigma$ bounds; a variation of approximately $\pm 2.5 \%$ is observed. This $\pm 2.5 \%$ variation corresponds to approximately a $1.5-\sigma$ profile from the GRAM- 95 variations. For altitudes above $34 \mathrm{~km}$, a search is underway to determine if measurement data is available. However, an estimate of the density that must have occurred above $34 \mathrm{~km}$ is presented in the Trajectory Reconstruction Section.

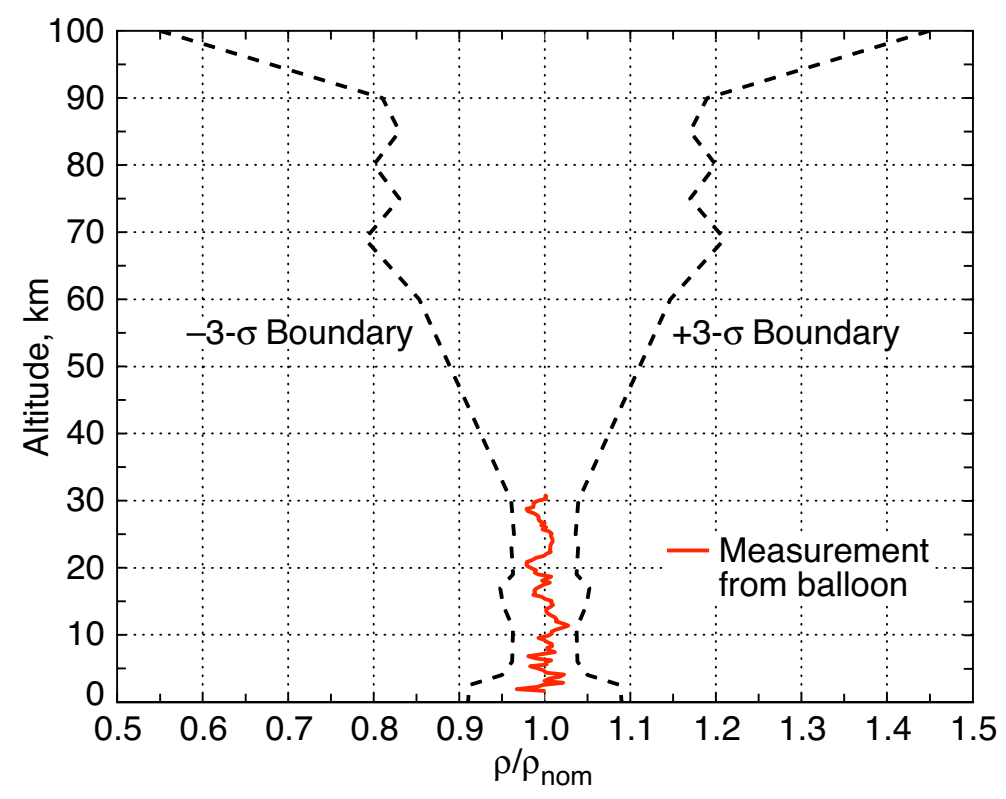

Figure 3. Density comparison to GRAM-95 model 


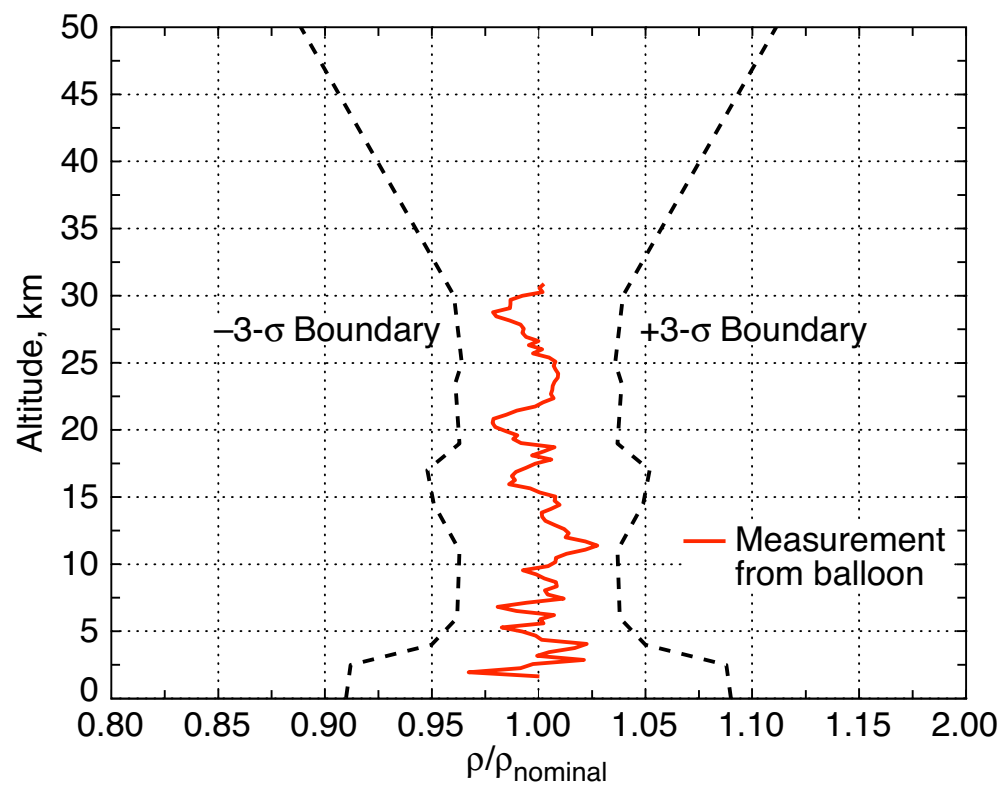

Figure 4. Density comparison to GRAM-95 model (expanded view)

Similarly, Figs. 5 and 6 show balloon measured wind data for the northward and eastward wind components. Also shown are the nominal profiles produced by the GRAM-95 model, as well as the respective upper and lower 3- $\sigma$ boundaries. As seen, the balloon measured northward and eastward wind components both fall well within the upper and lower 3- $\sigma$ bounds produced by the GRAM-95 model. The measured northward wind component varied between $\pm 5 \mathrm{~m} / \mathrm{sec}$ throughout the altitude band, and was lower in magnitude than the nominal profile produced by GRAM-95. The measured eastward wind component shows a sustained wind speed to the East similar to the nominal profile obtained from GRAM-95. However, the measured wind speed at $12 \mathrm{~km}$ (altitude of the jet stream) was higher than the nominal profile having a magnitude of approximately $27 \mathrm{~m} / \mathrm{sec}$ to the East. This measured eastward wind component corresponds to approximately a $1.5-\sigma$ profile from the GRAM- 95 variations. 


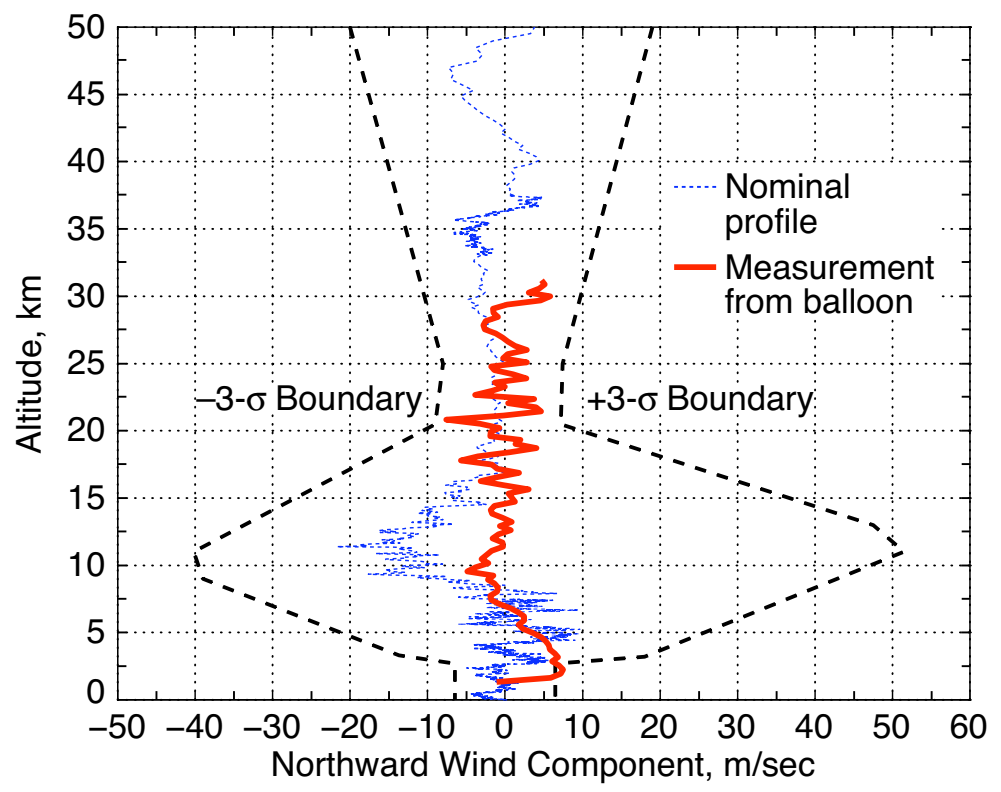

Figure 5. Northward wind component comparison to GRAM-95 model

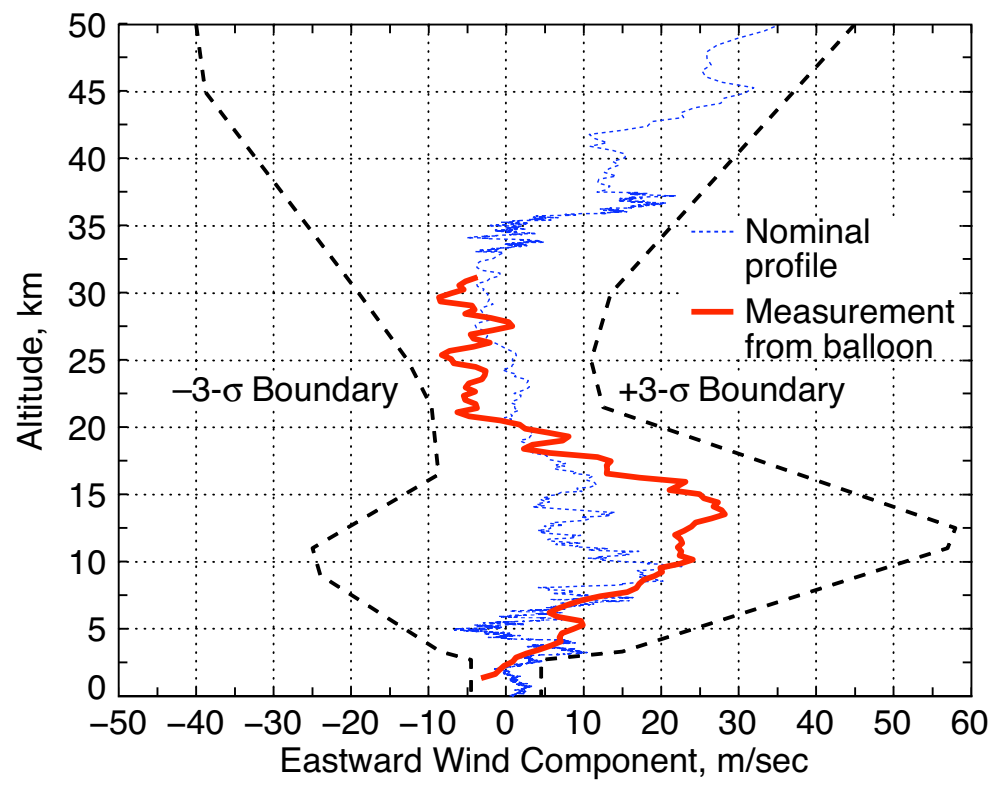

Figure 6. Eastward wind component comparison to GRAM-95 model

\subsection{Video Analysis of Tumbling Capsule}

Since there was no on board sensor data from which to perform an attitude reconstruction for the capsule during entry, video footage obtained from the UTTR radar tracking stations was employed to assess the onset of the capsule tumble. This section describes the video analysis that was performed. 
Several visible and infrared cameras that are a part of the UTTR infrastructure videotaped the Genesis capsule descent and impact. Videotapes from all of the cameras were reviewed to determine whether they would provide insight into technical details of the capsule's flight. Two videotapes were selected for analysis, one from an infrared video camera and the other from a visible light (color) video camera. Both cameras were actively tracking the capsule and each had an uninterrupted view for the final four minutes of flight. The two videotapes were recorded with different reference timing signals. However, they all were synchronized with each other, as well as other UTTR range data, using the impact event as the common reference. Time depicted in the figures for the video analysis is arbitrary and arises from the video digitization process. Also shown are the capsule descent Mach (M) numbers obtained from the UTTR radar tracking station data.

Software was developed to locate the capsule in the video frame and measure its total infrared luminance as recorded by the video camera. The video signal was recorded at 29.97 frames per second. However, each frame was composed of "fields" which were the even and odd raster lines of the frame. The video fields were recorded at twice the frame rate, or 59.94 fields per second. The software used to recover the luminance information measured the data on a per-field basis and, therefore, produced data at $59.94 \mathrm{~Hz}$.

The extracted luminance data is shown in the top plot of Fig. 7. There is a high frequency component in the data that is primarily above $20 \mathrm{~Hz}$. This component of the signal can be attributed to a number of sources of noise inherent in the use of analog video equipment. When the high frequency noise is removed (lower plot), the underlying variation of the capsule's luminance is more obvious. For the purpose of this analysis, the variation of luminance is assumed to correspond to capsule attitude motions that change the area of the forebody that is visible to the camera. There was no attempt to correlate magnitude of the luminance variation with capsule attitude. However, the observed frequencies of the luminance variation should correlate with natural frequencies predicted by pre-entry simulation predictions of the capsule attitude dynamics.

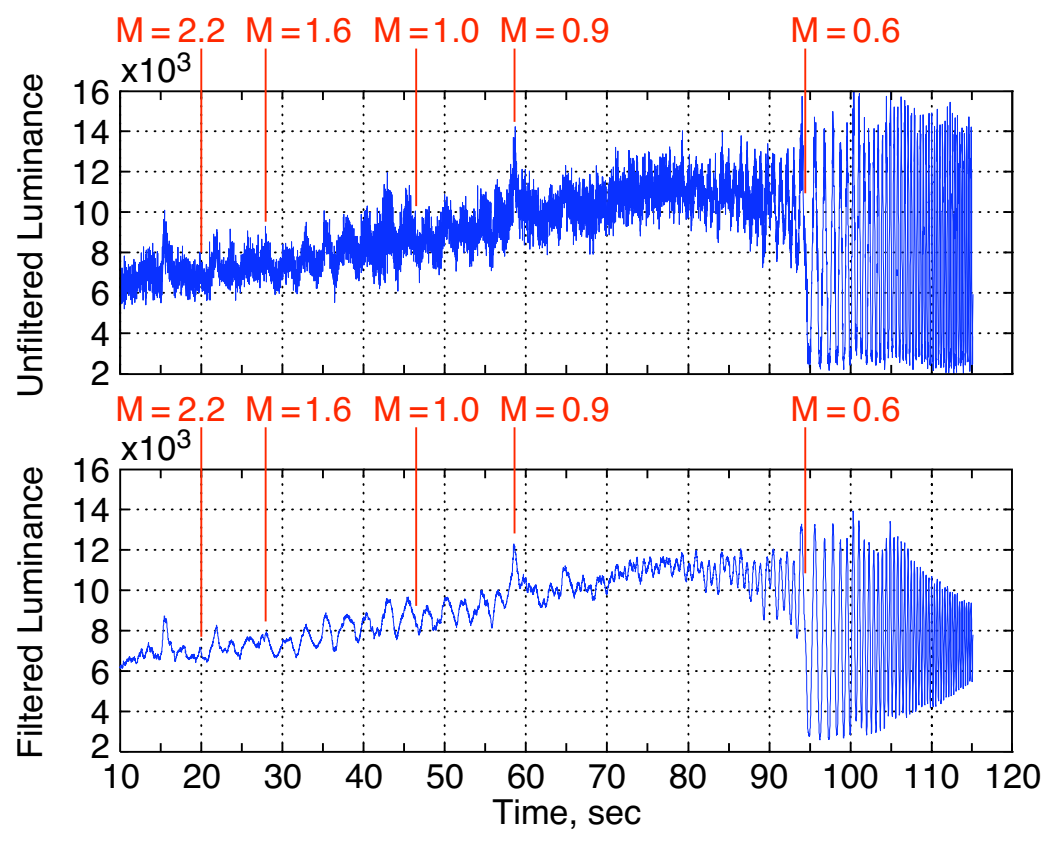

Figure 7. Unfiltered and filtered infrared luminance 
The frequency content of the extracted luminance data was analyzed using a Fast Fourier Transform analysis. The results are shown in Fig. 8, which depict the variation over time of the frequency distribution in the infrared signal. Between 20 and $55 \mathrm{sec}$, the dominant frequency is $0.42 \mathrm{~Hz}$. This frequency oscillation is also clear in the lower plot in Fig. 7. This oscillation is undetectable to the naked eye due to the low frequency and the large amount of noise in the video signal.

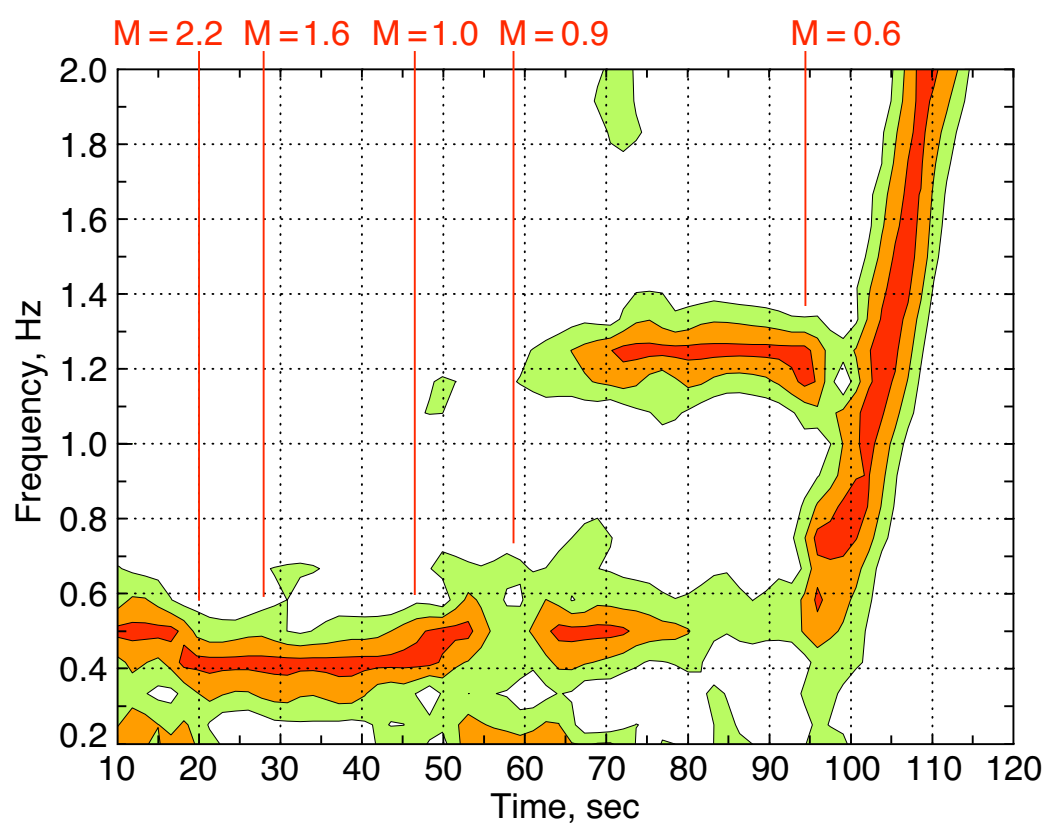

Figure 8. Frequency contours of luminance signal

At $59 \mathrm{sec}(\mathrm{M}=0.9)$, there is an excursion in the luminance data that interrupts the $0.42 \mathrm{~Hz}$ oscillations that had dominated the signal for the previous $30 \mathrm{sec}$. Following this peak in brightness, the $0.42 \mathrm{~Hz}$ signal is again apparent, but a $1.25 \mathrm{~Hz}$ signal is present and increases until it begins to dominate at $72 \mathrm{sec}$. This higher frequency continues to dominate until $95 \mathrm{sec}(\mathrm{M}=0.6)$, when the infrared video shows a target that is clearly tumbling with an increasing rate.

When examining the visible wavelength video at several times between 60 and $80 \mathrm{sec}$, the capsule appears to be tumbling, even though it is still small and faint within the frame. At roughly $80 \mathrm{sec}$, the brightness settings of the camera change making the tumbling unmistakable, because the dark forebody and white aftbody alternately come into view at a frequency that matches the $1.25 \mathrm{~Hz}$ observed in the infrared wavelength video.

Since the $0.42 \mathrm{~Hz}$ signal in Fig. 8 persists after the excursion at $59 \mathrm{sec}$, the appearance of the $1.25 \mathrm{~Hz}$ signal indicates a new mode that is superimposed on the previous $0.42 \mathrm{~Hz}$ capsule motion. Over the next 10 sec, the higher frequency motion begins to dominate the capsule's dynamics and continues to do so beyond $90 \mathrm{sec}$. The appearance of this higher $1.25 \mathrm{~Hz}$ frequency is interpreted as being the onset of tumbling. The time of this event corresponds to a Mach number of 0.9 when the video signal timeline is correlated with the UTTR radar tracking station data. 


\subsection{Aerodynamics Assessment}

The results from the video analysis, in conjunction with the use of the trajectory simulation [4] employed for the pre-entry predictions, can be used to corroborate the capsule aerodynamic database in the supersonic regime (between Mach 2.2 and 1.0).

Plotted in Fig. 9 is the capsule angle-of-attack history from the trajectory simulation without the deployment of the drogue parachute. Note, specific Mach numbers are highlighted. As the Mach number decreases, the capsule angle-of-attack increases from a few degrees at Mach 2.2 to very large angles at Mach 0.9 before tumbling in the simulation shortly thereafter at Mach 0.85 . This value of Mach 0.85 for the onset of tumbling from the simulation compares well with the Mach 0.9 estimate from the video analysis.

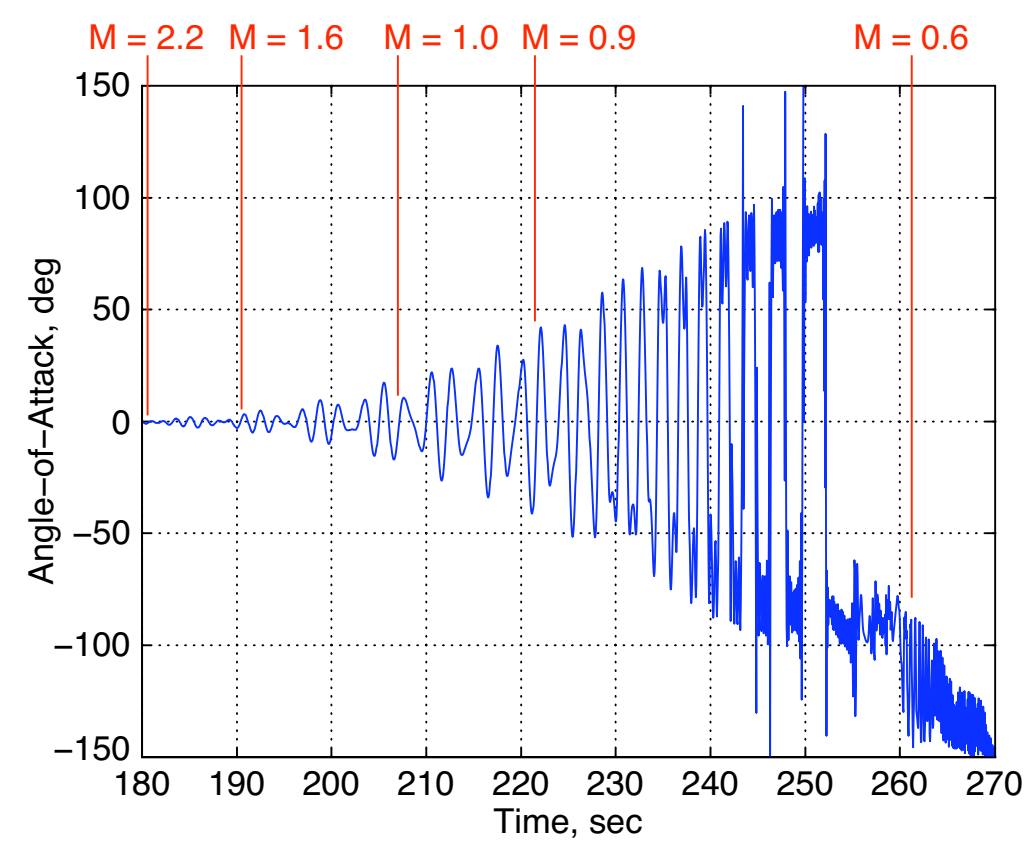

Figure 9. Time history of angle-of-attack from simulation

Figure 10 shows the comparison of the capsule deceleration, in terms of Mach number, versus time for the trajectory simulation data and tracking data obtained from the UTTR radar tracking stations. Note, the timelines of the trajectory simulation and the tracking data were aligned at Mach 1.0. A very good agreement is observed. This good agreement indicates that the aerodynamic database accurately captures the drag of the Genesis capsule from Mach 2.2 down to Mach 0.9. For these deceleration profiles to agree across the Mach range visible to the ground tracking stations suggests that the capsule was closely following the pre-entry predicted trajectory. 


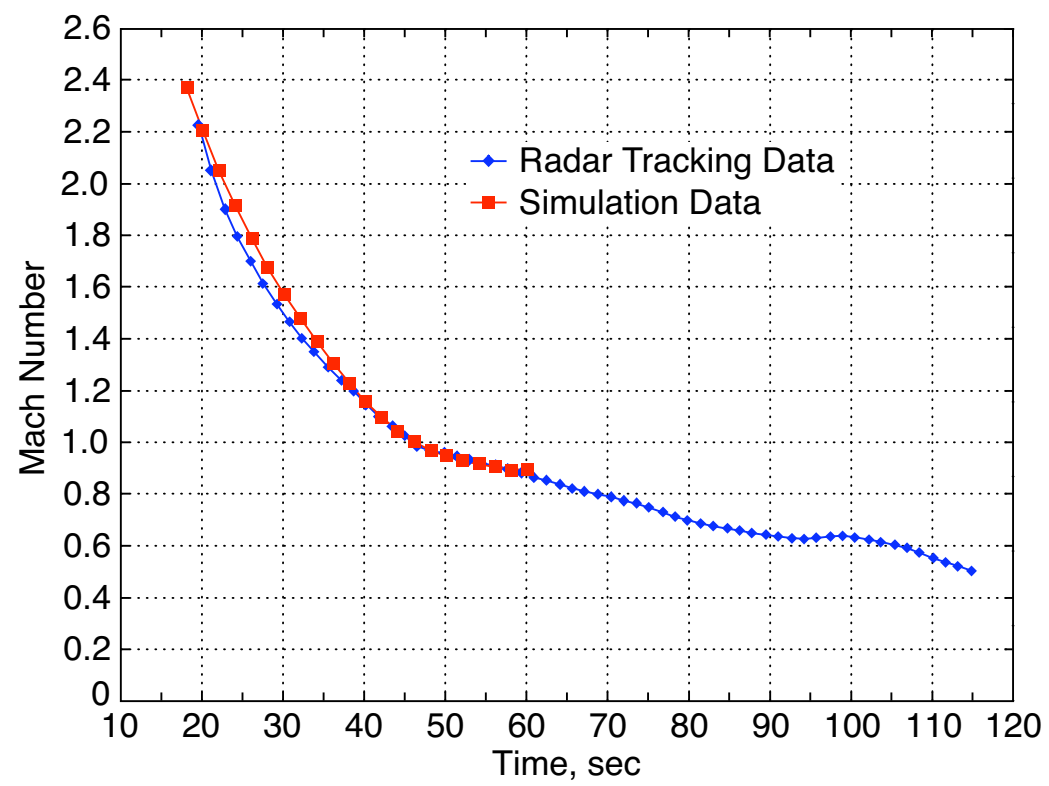

Figure 10. Mach number comparison

Figure 11 shows a comparison of the frequency content of the simulated angle-of-attack data of Fig. 9 and the measured infrared data presented in Fig. 8. Again, the timelines of the measured infrared signal and the trajectory simulation were aligned at Mach 1.0. As seen, the dominant frequencies correlate well over the range of Mach numbers where both data are present.

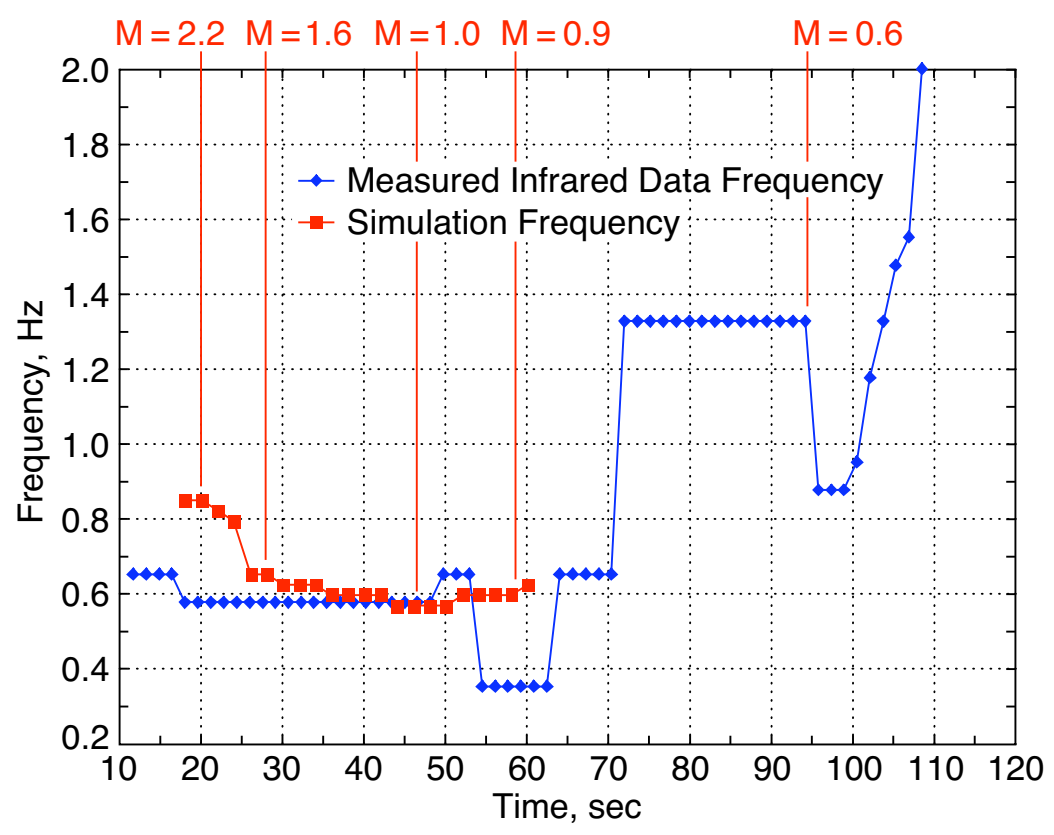

Figure 11. Frequency comparison 


\title{
DEEP IMPACT COMET ENCOUNTER: DESIGN, DEVELOPMENT, AND OPERATIONS OF THE BIG EVENT AT TEMPEL 1
}

\author{
Steven Wissler, Jennifer Rocca, and Daniel Kubitschek \\ Deep Impact Flight Team \\ Jet Propulsion Laboratory, California Institute of Technology \\ JPL M/S 301-490 \\ 4800 Oak Grove Drive \\ Pasadena, CA 91109 \\ Phone: (818)354-8649 \\ Fax: (818)393-6871 \\ Email: Jennifer.M.Rocca@jpl.nasa.gov
}

\begin{abstract}
Deep Impact is NASA's eighth Discovery mission. This low-cost, focused planetary science investigation gathered the data necessary to help scientists unlock early secrets of our solar system. The comet encounter with Tempel 1 was a complex event - requiring extremely accurate timing, robustness to an unknown environment, and flight team adaptability. The mission operations and flight systems performance were spectacular for approach, impact, and lookback imaging on July 4, 2005.

The Encounter Phase began one week prior to impact with a strenuous imaging campaign, including mission critical optical navigation and instrument calibrations, plus imaging for coma characteristics and nucleus rotation. Until just prior to impact, the comet nucleus was unresolved spatially, making operations demanding in the final days. Flyby and Impactor separation occurred 24 hours before impact, beginning the most intense part of DI's mission. The independent, yet coordinated missions of the two spacecraft were accomplished with elegant sequence design, an ambitious fault protection and recovery strategy, and a battery of punishing tests performed with very few resources to retire risk for the encounter event.

Encounter Approach imaging and calibrations were successfully executed, giving the science and navigation teams an array of early images in preparation for impact. The final Trajectory Correction Maneuver, Autonomous Navigation, and Attitude Control operations achieved desired performance during encounter - enabling stunning images captured as the Impactor approached Tempel 1, and as the flyby witnessed the impact and subsequent departure of the comet. The key strategy for data management, "live for the moment," was employed as planned; all high priority images were transmitted to the ground prior to closest approach.
\end{abstract}

Despite engineering challenges, an unknown and potentially dangerous comet environment, and a short operations phase, Deep Impact was a dramatic success. This Discovery mission spent Independence Day with the world watching-what a sight! 


\subsection{INTRODUCTION}

The Deep Impact mission explored the interior of Comet Tempel 1 by using a 364-kg Impactor to excavate a crater in the comet's surface and taking data on the newly-exposed cometary interior with a companion flyby spacecraft. Deep Impact is the eighth mission in NASA's Discovery Program, following NEAR, Pathfinder, Lunar Prospector, Stardust, Genesis, CONTOUR, and MESSENGER. The project was organized as a team between the principal investigator, Dr. Michael A'Hearn of the University of Maryland; the science team of eleven other prominent experts on comets, remote sensing, and impact physics; the industrial partner, Ball Aerospace \& Technologies Corp.; and the Jet Propulsion Laboratory as the NASA lead center. ${ }^{1}$

This paper will give an introduction to the Deep Impact mission, with special focus on the comet encounter event. The design approach to this multi-dimensional activity will be discussed, delving in to key elements contributing to the complexity of encounter design. The comprehensive test program implemented to verify and validate the design will also be addressed, including a summary of encounter performance predictions made at its completion. Finally, results of Deep Impact's encounter with Tempel 1 will be shown, focusing on the engineering performance throughout the final mission phase.

Deep Impact's low-cost, focused planetary science investigation gathered the data necessary to help scientists unlock early secrets of our solar system. The comet encounter with Tempel 1 was a complex event - requiring extremely accurate timing, robustness to an unknown environment, and flight team adaptability. The mission operations and flight systems performance were spectacular for approach, impact, and lookback imaging on July 4, 2005.

\subsection{Mission Synopsis}

The Deep Impact mission conducted a scientific cratering experiment on the nucleus of comet Tempel 1 near the time of perihelion for its 2005 apparition. This was accomplished by launching two joined spacecraft January 12, 2005 to approach the comet in early July 2005. The short six month cruise phase was comprised of a battery of engineering checkout/calibration activities (commissioning), and in-flight demonstrations of autonomous navigation and encounter-like imaging. Two course maneuvers were conducted during cruise to refine the path to Tempel 1. Figure 1 shows the flight system.
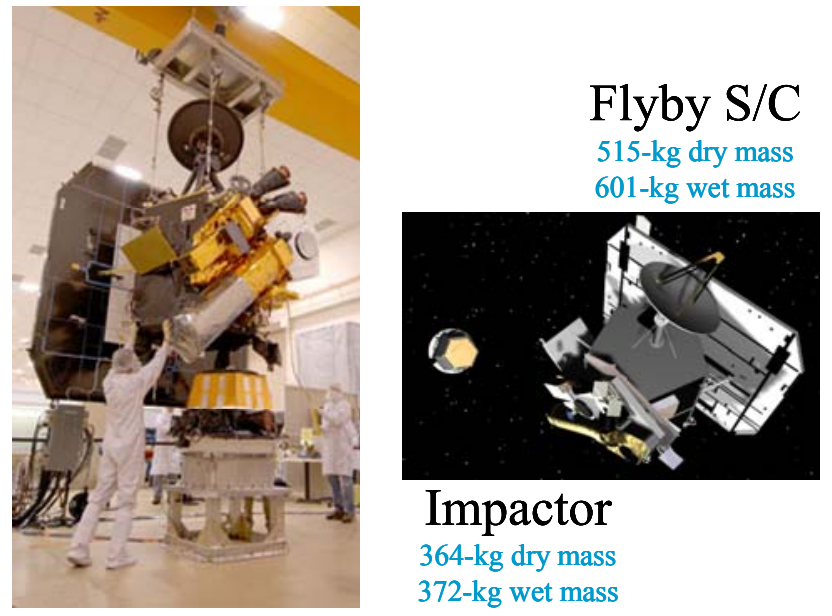

Measured Flight System Mass: 972.93 kg

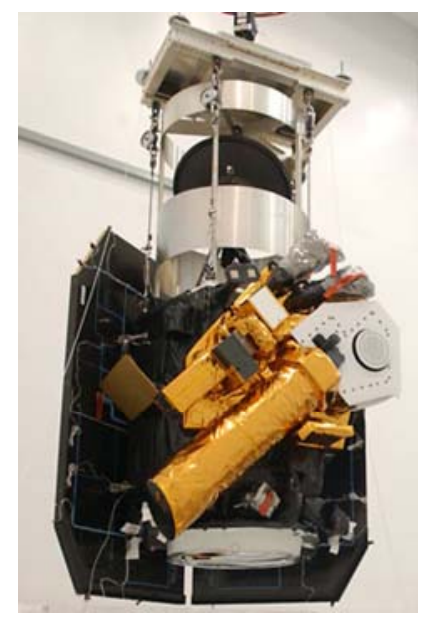

\section{Figure 1: Deep Impact Flight System}

Encounter Approach began one week prior to impact with a strenuous imaging campaign, including mission critical optical navigation and instrument calibrations, plus imaging for coma characteristics and nucleus rotation. Until just prior to impact, the comet nucleus was unresolved spatially, making operations demanding in the final days. Using spacecraft optical observations of the comet and conventional ground-based navigation techniques, the joined spacecraft were maneuvered as close as possible to a collision trajectory with the nucleus of Tempel 1 , and the Impactor was released 24 hours before impact. 
The Impactor observed the approaching nucleus with an optical camera and maneuvered itself to a collision course toward the lighted portion of the nucleus. After separation from the Impactor, the flyby spacecraft performed a maneuver to delay and deflect its flight path toward the nucleus so that it could observe the impact, ejecta, crater development, and crater interior during a 500-km flyby of the nucleus that occurred about 14 minutes after the impact. The flyby spacecraft carries a remote sensing payload of two instruments for imaging and infrared spectroscopy. Close-in observations of the nucleus by the Impactor camera were sent to the flyby spacecraft by an Sband radio link in the last seconds before impact. Simultaneous observations of the comet before, during, and after the impact were also conducted from ground and space-based observatories as an essential part of the total experiment. All scientific and supporting engineering data are archived for future use by the scientific community. Figure 2 shows an overview timeline for the mission.

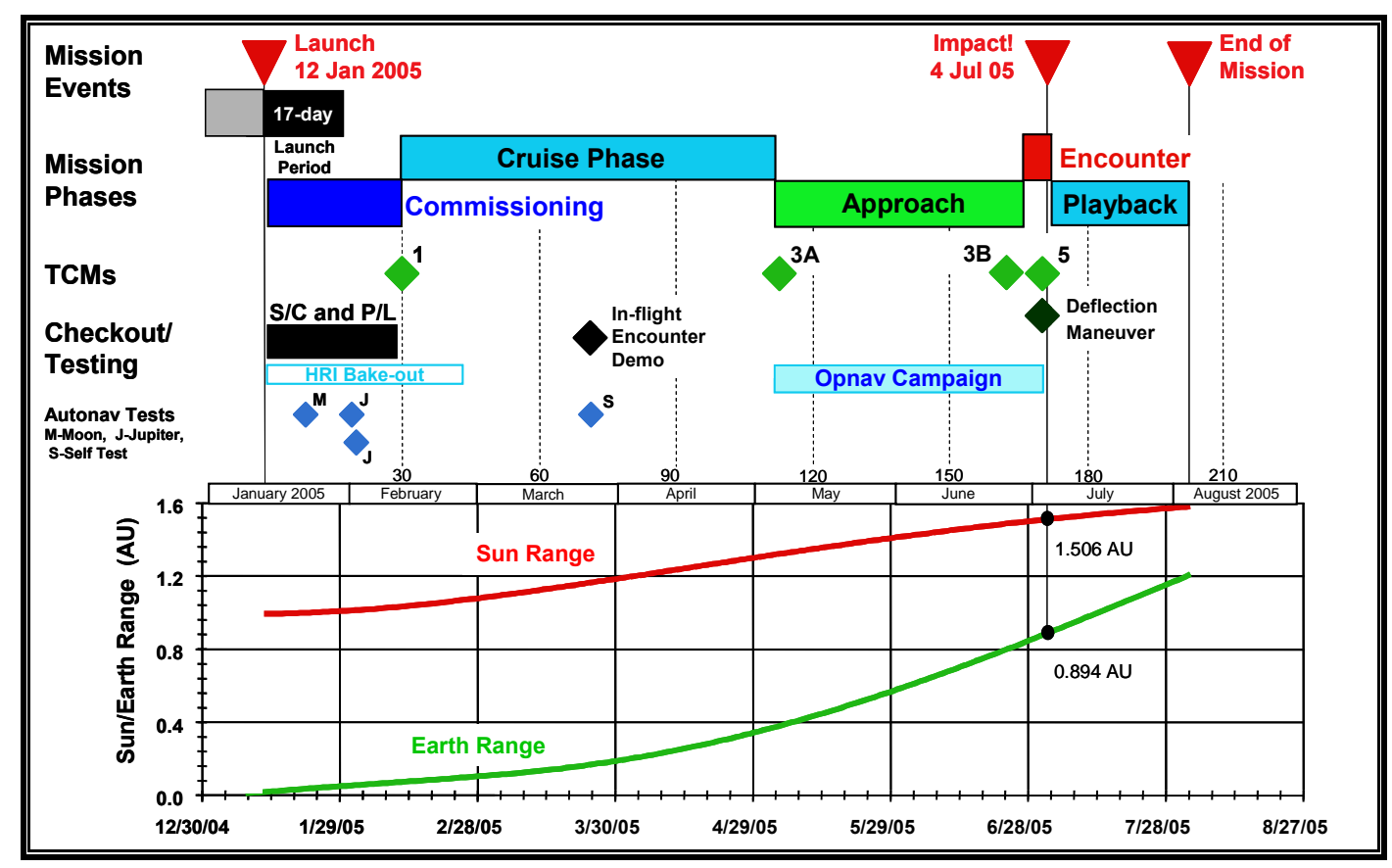

Figure 2: Deep Impact Mission Timeline

\subsection{Mission Objectives}

From the Discovery Program Plan, Deep Impact's Primary Mission Objectives:

- Dramatically improve the knowledge of key properties of a cometary nucleus and, for the first time, assess directly the interior of a cometary nucleus by means of a massive Impactor hitting the surface of the nucleus at high velocity.

- Determine properties of the surface layers such as density, porosity, strength, and composition from the resultant crater and its formation.

- Study the relationship between the surface layers of a cometary nucleus and the possibly pristine materials of the interior by comparison of the interior of the crater with the pre-impact surface.

- Improve our understanding of the evolution of cometary nuclei, particularly their approach to dormancy, from the comparison between interior and surface.

Deep Impact employed the comet encounter with Tempel 1 to address these objectives.

\subsection{Comet Encounter Overview ${ }^{2}$}

Critical comet encounter activities began $24 \mathrm{hrs}$ prior to the expected time of impact (TOI), when the two spacecraft separated. The encounter geometry resulted in an illumination phase angle of approximately $65^{\circ}$ for the Tempel 1 nucleus and induced some self-shadowing due to the shape and orientation of the nucleus. The Flyby s/c performed a slowing maneuver with a $\Delta \mathrm{V}$ of approximately $102 \mathrm{~m} / \mathrm{s}$ to provide $800 \pm 20 \mathrm{sec}$ of post-impact event imaging and 
control the flyby miss-distance to $500 \pm 50 \mathrm{~km}$ (chosen to minimize damage to the flyby spacecraft while still obtaining high resolution impact images). During the first $22 \mathrm{hrs}$ following release, the Impactor s/c acquired and telemetered science and navigation reconstruction images to the ground using the Flyby s/c as a bent-pipe relay. The Flyby s/c also acquired and telemetered MRI and HRI visible and HRI infrared (IR) images of the nucleus and coma. Figure 3 shows a schematic diagram of the encounter activities.

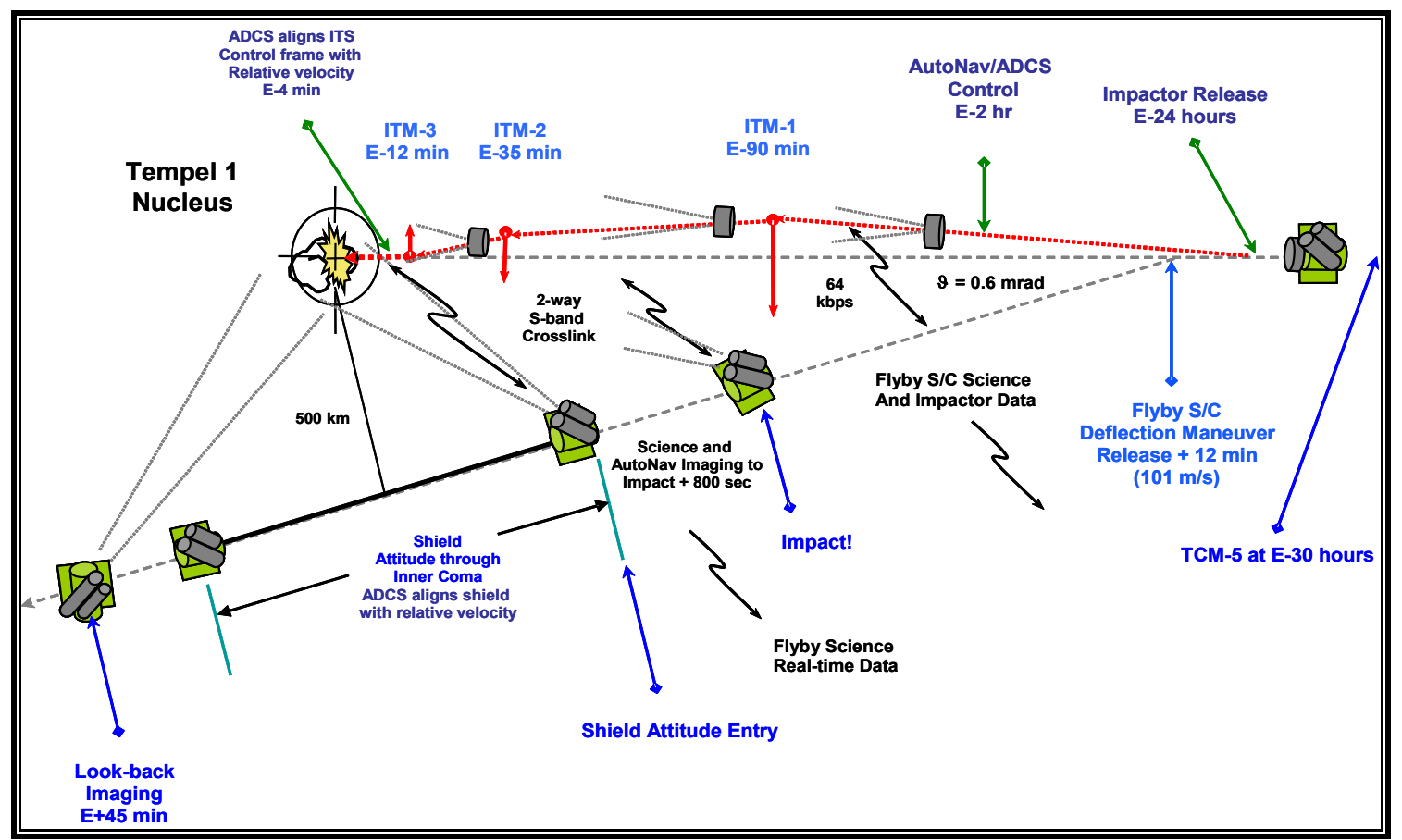

Figure 3: Comet Encounter Schematic

The autonomous phase of the encounter began at $120 \mathrm{~min}(2 \mathrm{hrs})$ before TOI, when the comet nucleus could be resolved by the impactor instrument. A critical sequence running on-board the Impactor spawned a science and navigation subsequence that issued Impactor Targeting Sensor (ITS) commands to produce navigation images at a $15 \mathrm{sec}$ interval. The Autonomous Navigation (AutoNav) software (originally developed and demonstrated during the Deep Space 1 (DS1) mission) processed these images to form observations for the purpose of trajectory determination (OD). OD updates were performed every minute. Three Impactor Targeting Maneuvers (ITMs) were computed by AutoNav and executed by the Attitude Determination and Control System (ADCS): ITM-1 at E-90 min, ITM-2 at E-35 min, and ITM-3 at E-12 min. At E-2 min, the Impactor ADCS pointed the ITS along the AutoNav estimated comet-relative velocity vector to capture and telemeter high resolution $(20 \mathrm{~cm})$ ITS images of the impact site prior to impact. Meanwhile, the AutoNav software on the Flyby s/c was processing MRI images of the comet every $15 \mathrm{sec}$ and updating the trajectory of the Flyby s/c every minute to continuously point the MRI and HRI instruments at the nucleus.

\subsection{COMET EnCounter Design}

The following sections will highlight major design elements of the flyby spacecraft's comet encounter. To introduce the design, driving requirements will be shown. The section will continue with the encounter timeline, depicting a complex integration of science and engineering activities. Each of the major design elements of encounter will also be discussed, including Separation/Divert and Final Flyby Sequence Timing Design, Data Return Strategy, Imaging Campaign, Autonomous Navigation, and Robustness. Each of these elements was central to the successful Tempel 1 encounter design.

\subsection{Design Envelope}

The Deep Impact encounter phase was very complex for a low-cost Discovery mission. The mission requirements and cost trades which drove the design of the encounter operations were: 
$\square \quad$ Live for the moment science return: There was a significant chance that the Flyby spacecraft would not survive the cometary dust environment at the closest approach to Tempel 1 . The primary science data had to be transmitted by the spacecraft prior to the time of closest approach. This requirement drove the design of the telecom subsystem, requiring higher-power amplifiers and a larger high-gain antenna.

- High resolution crater imaging: This requirement increased the accuracy requirements for attitude control (precision and stability) and necessitated the use of on-board autonomous navigation on the Flyby spacecraft.

$\quad 30 \mathrm{~km}$ Impactor release accuracy: The requirement to release the Impactor at 24 hours prior to impact with a B-plane accuracy of less than $30 \mathrm{~km}$ required a trajectory correction maneuver (TCM) to be done 6 hours prior to Impactor separation and with a tracking data cut-off 11 hours prior to maneuver execution.

$\square \quad$ On-board autonomous fault protection and critical sequence resumption. This level of fault protection and recovery was far more complex than on other discovery class missions and was difficult and costly to test.

$\square$ De-scopes to the flight system due to cost increases also drove up operational complexity. Some examples:

- On-board file system space was cut in half. This increased the complexity and frequency of on-board file management. The backup flight computer file storage changed from being entirely redundant storage to storing some amount of unique images.

- The communication link between the two flight computers was descoped, affecting the ability of fault protection software to autonomously recover from a flight computer swap. In the event that a swap occurred, the re-configuration of the spacecraft and re-starting the execution of the encounter sequence would have required ground-in-the-loop.

- Downlink data rate was de-scoped from 400,000 bps to 200,000 bps. This descope increased the contention for bandwidth for science images, AutoNav images and Impactor telemetry files, significantly increasing the complexity of the sequence design and testing.

\subsection{Encounter Activity Timeline}

For the majority of the mission, Tempel 1 was smaller than one pixel across in all of the Deep Impact imagers. Only a few weeks prior to impact did the comet start to grow in size appreciably. Real preparations for encounter, including targeting and final imaging calibrations, had to be conducted very close to the critical event. This resulted is the last week prior to encounter being one of the most challenging of the mission due to the vast scope of activity required so close to the impact. The Encounter Phase began with a week of Encounter Approach activities to prepare for final critical operations. Key elements of approach included: activation of the critical encounter sequence, maneuvering to continuous comet-pointing attitude, extensive optical navigation and science imaging sequence execution, divert maneuver design and uplink, final science point spread function and infrared mini-calibrations, Trajectory Correction Maneuver design and uplink, and final preparation of the Impactor spacecraft for separation and its subsequent free-flight to Tempel 1. In addition to these major elements, regular maintenance activities were also performed during approach, including regular science and navigation image downlinks, ITS imaging and downlinks, delta-DOR, and Impactor engineering telemetry playback.

All encounter approach activities were designed to execute on-board via sequences, with the only ground commands issued to kick off the backbone sequence. This design decision was intended to reduce operational burden on the flight team so near to impact. Continuous 70-m antenna coverage and nearly continuous 34-m antenna back-up coverage provided by the Deep Space Network were required to support Deep Impact's encounter. During the final week, both spacecraft were fully powered, with all instruments and engineering subsystems in operation. This week represented the first extended period of continuous Impactor spacecraft operation outside of checkout/calibration/demonstration activities performed during cruise.

It was discovered during early commissioning that Deep Impact's High Resolution Imager was out of focus. The science team devised a plan to use deconvolution on images taken with this telescope. In order to perform successful deconvolution, a set of mini calibrations needed to be performed within 48 hours of impact. These calibration images were labeled as critical to mission success, as they would enable the science team's ability to post-process the HRI images and achieve improved resolution compared to the out-of-focus images.

Activity design for this mission phase was severely constrained. For the majority of the entire week, nearly every spacecraft subsystem was required to function at capacity. Systems engineering of the integrated activity relied on iterative testing, intense modeling of the data flow, and innovative sequence design. The final sequence designs for 
Encounter Approach and Encounter, represented by the timeline in Figure 4, were predicted to meet all constraint limitations.

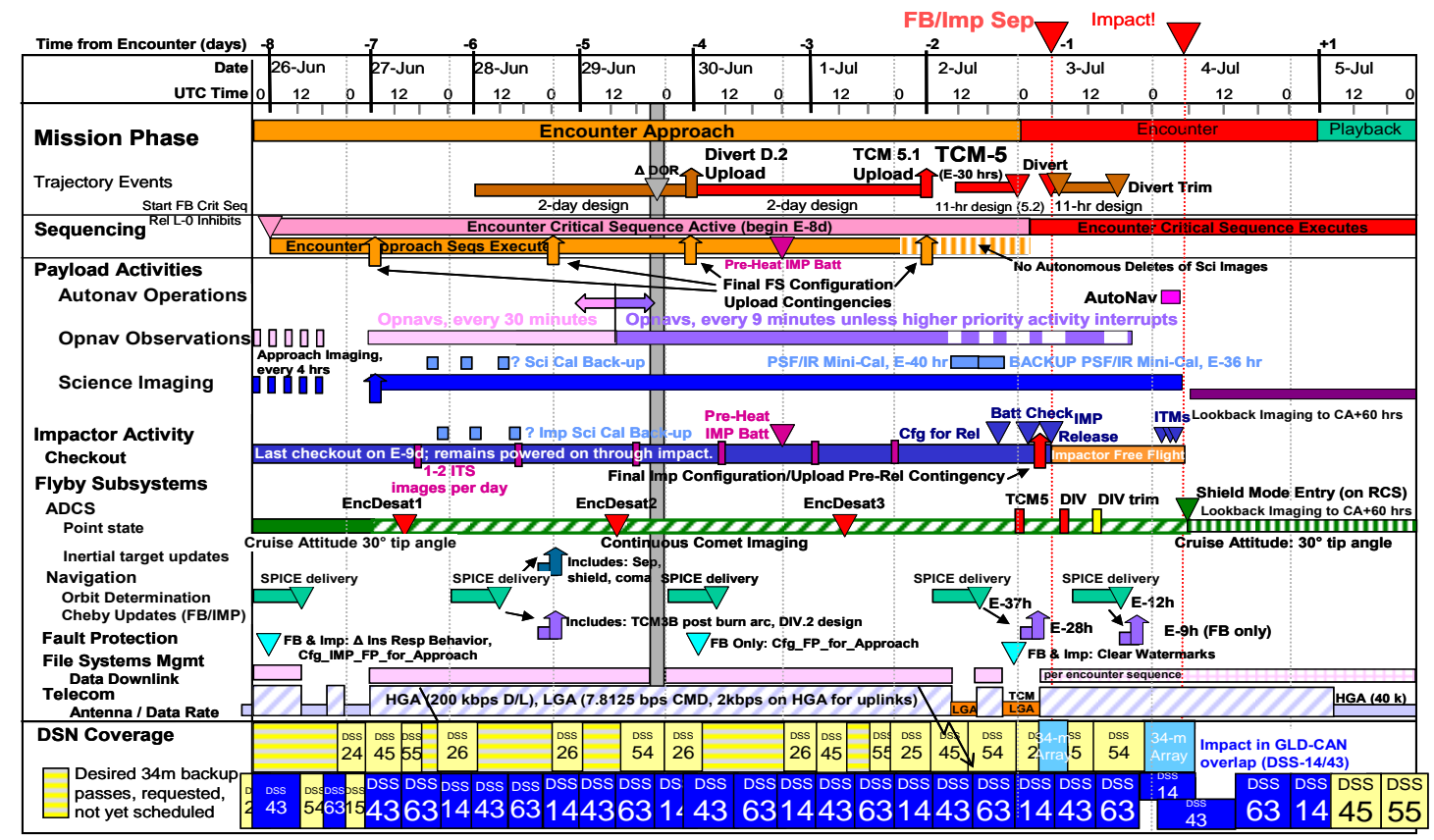

Figure 4: Encounter Phase Timeline

The most challenging encounter elements, from an activity and sequence design standpoint, are discussed in the following sections.

\subsection{Separation/Divert Sequence Timing Design}

Separation was the first encounter event designated as critical. The release of the Impactor from the Flyby happened only 24 hours prior to impact, to allow the impactor the best chance of seeing the nucleus of the comet and allow time for the flyby to divert its course. Separation entailed 'launching' the Impactor, then letting it fly alone for only one day prior to its main event. To place the impactor on an initial impact trajectory, the flight system was targeted to impact the comet with a propulsive maneuver 6 hours before separation. The Flyby spacecraft needed to perform a propulsive Divert maneuver of 101 meters/sec 12 minutes after Impactor separation in order to slow down and miss the comet by 500 kilometers as planned. This slow down was required in order for the Flyby to observe the impact and crater formation for 13 minutes prior to closest approach.

Design implications resulting from Separation/Divert included precise sequence timing design and an operations concept involving two independent spacecraft teams for separate Flyby and Impactor operations. In addition, due to the critical nature of Impactor release, its proximity to the actual encounter, and the long separation between the spacecraft and Earth (light time), the spacecraft had to be able to autonomously recover from a fault during separation and divert and re-start the on-board sequence. To implement this, the sequence used a FSW mark and rollback capability, where the sequence could be re-started, and rolled back to a previous event, or rolled forward to the next event. The separation and divert sequences were a set of relative-timed Sub-sequences activated by the absolute-timed Flyby Critical Sequence. The separation and divert sequences were broken down into fragments which facilitated fault protection's ability to respond to a fault during separation and divert by interrupting the sequence, fixing the fault, and resuming the sequences. The break points in these sequences were carefully chosen to accommodate all imaging, fault protection, timing, and data flow constraints. The final separation sequence design is shown in Figure 5. After Divert, the next critical and equally challenging activities were the flyby and look back imaging events, discussed in the next section. 


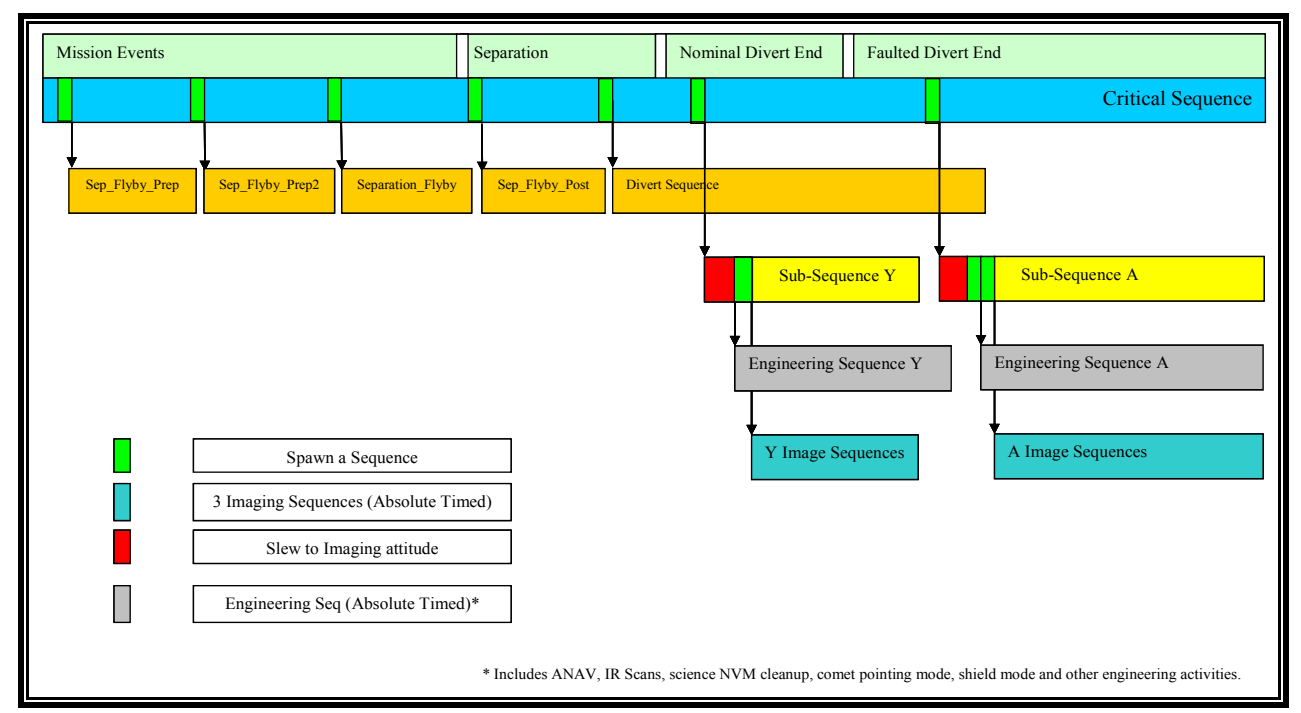

Figure 5: Separation/Divert Sequence Design

\subsection{Flyby Sequence Timing Design}

After Divert, the flyby periodically imaged the comet and sent these images and Impactor telemetry to the ground. Two hours prior to impact, the flyby began Autonomous Navigation (AutoNav) operations (discussed in section 2.7), which increased the frequency of imaging. The E-2hr timeframe for AutoNav was selected to coincide with the Impactor AutoNav timeframe, which was selected to allow for trajectory correction of delivery error up to $30 \mathrm{~km}$ while leaving enough propellant for all three ITMs. AutoNav images were generated at a rate of four per minute, with orbit determination (OD) updates once per minute. When the dust environment was predicted to become more intense, the flyby's ADCS transitioned to an attitude mode (Shield Mode) which kept the solar array edge in the direction of the anticipated comet dust flow. Shield Mode allowed the dust shield to protect the solar arrays and instrument electronics. This transition was timed to occur when the Flyby spacecraft was $700 \mathrm{~km}$ from Tempel 1 and 13 minutes 22 seconds after nominal Impactor impact. The Flyby spacecraft remained in this attitude for 27 minutes, until it was through the worst of the dust environment. Subsequently, it turned back to perform look back imaging of Tempel 1. In look back attitude, the High Gain Antenna couldn't be pointed at Earth. The look back imaging was performed on the Low Gain antenna at a downlink rate of 10 bits/second. The Flyby spacecraft was sequenced to periodically switch between an attitude which allowed for playback of stored images, and the look back imaging attitude. AutoNav operations, Shield Mode entry, and numerous flyby maneuvers, coupled with nearly continuous Science imaging of the comet nucleus, made sequencing the last hours of encounter the most challenging sequence design task of the mission.

In addition to intense imaging and flyby maneuvers, sequence timing synchronization with the impact event was also a major encounter design consideration. To ensure the flyby would capture images of the impact and subsequent cratering event, the high-rate imaging sequence for impact needed to be started with an accuracy of approximately 3 seconds. The final imaging sequence for the high-resolution crater imaging had a similar timing requirement. The flyby sequence design had to allow AutoNav (the system which determined TOI) to adjust the imaging sequence spawn command timing to enable the precise imaging sequences to start at the appropriate time. The a priori error in time of impact (TOI) was +/- 3 minutes, and Autonav could adjust when the final imaging sequence started by calculating a TOI and time of final imaging (TOFI) update. The TOFI update also controlled the timing of the transition to shield mode.

The final sequence design for encounter, similar to separation/divert, included relative-timed Sub-sequences spawned from the critical sequence. Figure 6 shows the sequence architecture for encounter, with break points implemented to accommodate all constraints and timing updates. The breaks just prior to impact and prior to the start of the Shield Mode sequences were chosen to accommodate the AutoNav timing updates. 


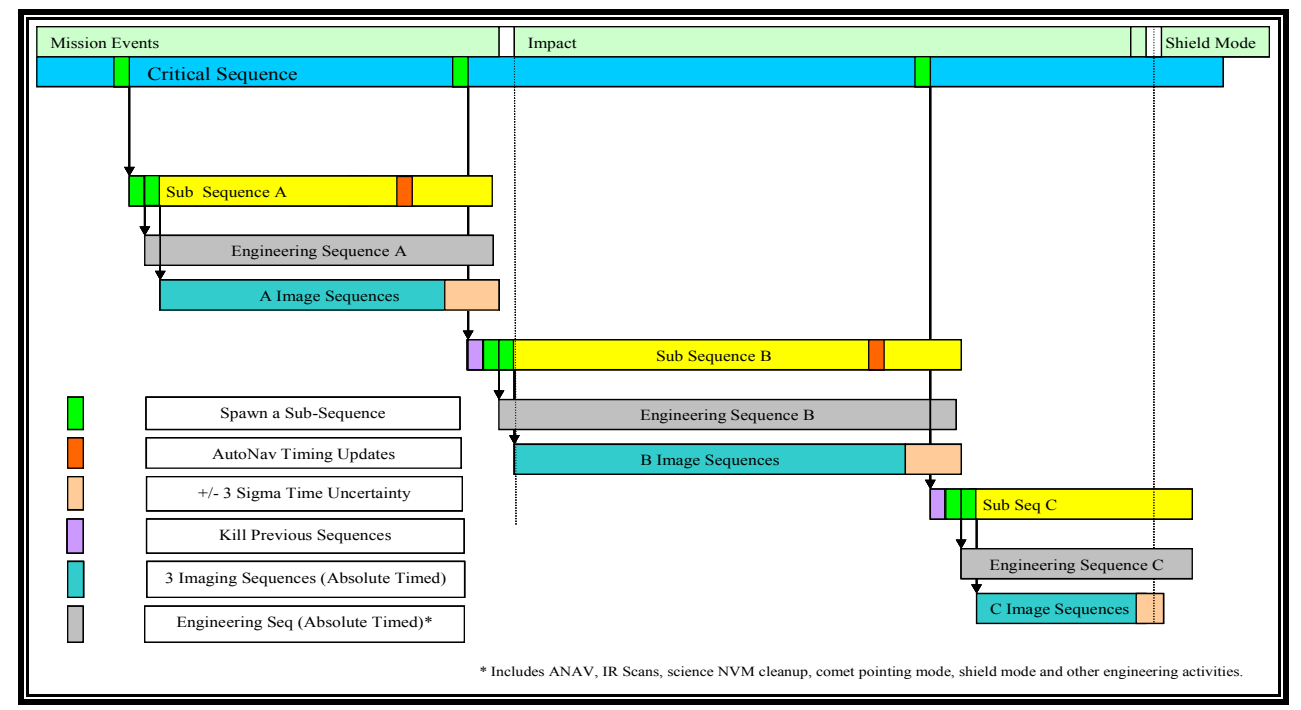

Figure 6: Encounter Sequence Design

\subsection{Data Return Strategy}

Another key design element of the Deep Impact comet encounter was the data return strategy. The storage, management, and downlink of all engineering and science data from both spacecraft was a major challenge in the design of an activity that could not just execute encounter, but could get data to the ground that proved the event successful. The hopeful destruction of the impactor spacecraft, and the possible damage to the flyby spacecraft required a specialized data flow prioritization approach. This section highlights the data flow considerations and final design at encounter.

The Deep Impact Flyby Spacecraft stored engineering telemetry and instrument image files on a file system in nonvolatile memory (NVM). There were two $256 \mathrm{MB}$ NVM devices available. The first device had 2 dedicated drives for Flight Software and parameter tables. This first device also had a general purpose data storage drive, which contained sequence and parameter files uplinked from the ground, all engineering telemetry files and "overflow" instrument image files. The second device was dedicated to instrument image files. Table 1 shows NVM Allocations for encounter.

Table 1: Encounter Flyby S/C NVM Allocations

\begin{tabular}{|l|l|}
\hline Data Type & Allocation \\
\hline Science & 309 MBytes \\
\hline Navigation & 6.95 MBytes \\
\hline Impactor Telemetry & 30 MBytes \\
\hline
\end{tabular}

The Instruments could send images to either or both of the flight computers. The backup flight computer's file system was primarily used for redundant storage, however, due to the descope in file system space, some images were written non-redundantly to one or the other flight computer in order to preserve some of the original science data. This significantly complicated the sequencing and data return strategy. During post-encounter playback, it was necessary to swap telemetry streams to the backup flight computer to retrieve images. Swapping telemetry streams was undesirable, as the backup flight computer did not have an active 1553 interface to the flight hardware controlling the spacecraft, thereby limiting visibility for the flight team during some playback cycles.

Instrument image files could be queued for downlink to the ground in real-time (via correct parameter in imaging command), or sent to the ground later (via a file downlink command). All images prior to Impact minus 5 hours were sent to the ground in real-time. These images were periodically sequenced for deletion prior to ground confirmation of receipt in order to clear NVM for new image acquisitions. Within 5 hours of impact, the image rate increased, as did the importance of those images. Due to the shift in priority, most flyby science images were kept in NVM storage until after the playback phase completed, and even at that time all images were confirmed received on the ground prior to deletion. NVM was nearly filled to capacity at shield mode entry, only minutes after impact. 
The look back imaging, which occurred following the flyby's closest approach to Tempel 1, used the remainder of the available NVM.

The Impactor spacecraft communicated with the Flyby spacecraft via an S-band radio. Commands and file transfers to the Impactor were processed on the ground into a file of bits to be radiated, then uplinked to the Flyby spacecraft as a file transfer to the Flyby's on-board file system. A command was then sent from the ground for the Flyby to send that file using the s-band link to the Impactor, which saw it as either a real-time command or a file transfer.

Telemetry data received on the Flyby from the Impactor S-Band was stored in $128 \mathrm{kB}$ NVM files and automatically queued up for $\mathrm{D} / \mathrm{L}$. When the files were received on the ground, they were automatically read and processed into Impactor telemetry frames for real-time display and storage. The collection and storage of this data on the Flyby could be turned on or off by command. There was sufficient data allocation on the Flyby NVM to store $30 \mathrm{MB}$ of Impactor telemetry. This equated to just over 1 hour of data at $64 \mathrm{Kbps}$. The Impactor data collection strategy was to turn on collection, collect data for 1 hour, turn off data collection and delete the files in NVM. This sequence was repeated until one hour before impact. The last hour was collected and remained in NVM post impact. This strategy ensured nearly continuous Impactor telemetry to the ground, but only stored the last hour of telemetry on the Flyby for post encounter playback. Any telemetry not received on the ground prior to 1 hour before impact was unrecoverable if initial transmission attempt failed. Due to descopes affecting downlink data rate, there was major contention between science image data and Impactor telemetry in the available downlink bandwidth. The sequences and downlink priorities had to be carefully orchestrated in order to avoid latency in either science images or Impactor telemetry.

Flyby S-Band RF reception was turned on at Separation plus 30 seconds. S-Band RF transmit was turned on at Separation plus 6 minutes to ensure the RF level was low enough to avoid damage to the Impactor S-Band receiver. In the final encounter sequence, it was estimated that over 6000 Impactor telemetry files (about $760 \mathrm{MB}$ ) would be collected by the Flyby and relayed to the ground during the encounter critical sequence.

The data flow was the hardest problem to solve in terms of design. C\&DH throughput performance was a continual problem throughout most of the encounter test program, with critical impact and crator images lost on nearly every test run on the spacecraft pre-launch and testbed runs pre- and post-launch. The throughput performance fell off from predictions during periods of heavy imaging and file system usage. All other subsystems showed excellent performance during testing long before the data flow models and testing predicted success. To come to a workable design, intense effort was spent throughout the test program to feedback improvements to the design. The performance was improved with Flight Software updates throughout the test program, and appeared adequate for flight in the final 2 months prior to encounter. Mission success depended on critical images being transmitted to the ground prior to spacecraft loss (impactor) or damage (flyby), and the final design, depicted in Figure 7, showed that all high priority images would be transmitted prior to closest approach as required.

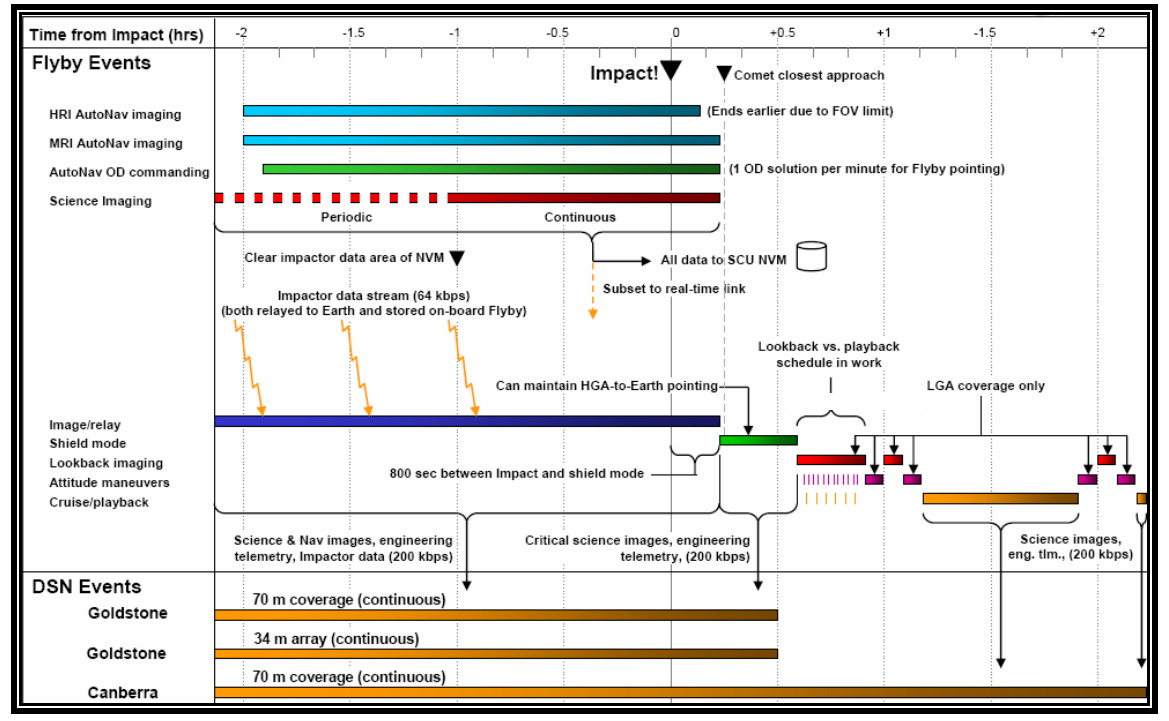

Figure 7: Encounter Flyby Spacecraft Data Flow Overview 


\subsection{Flyby Encounter Imaging Campaign}

The majority of commands in the encounter sequences were imaging commands. The imaging campaign for encounter was a critical element of the full design. The Science and Navigation Teams generated the absolute-timed imaging sequences which had to be integrated with the engineering sequences designed by the flight team. The encounter imaging campaign consisted of a combination of 6012 science, 199 optical navigation and 936 autonomous navigation images. Early encounter science imaging (prior to E-2 hours), was primarily dedicated to periodic monitoring of the comet, definition of the rotation, and detection of the nucleus. The nucleus of the comet was resolved at around 82 hours prior to impact in the HRI and 16.5 hours prior to impact in the MRI.

The transition between optical navigation and autonomous navigation occurred two hours before impact. Optical navigation images were used for determining the location of the comet relative to the spacecraft, in order to improve the Impactor delivery accuracy prior to the Impactor's autonomous navigation phase. The on-board autonomous navigation software took over 2 hours before impact and was used to keep the instruments pointed at the comet. The primary camera for AutoNav was the MRI, however, redundant AutoNav images were captured with the HRI as well. The redundant HRI images were discarded if MRI images were successfully acquired.

Science and navigation shared use of the same instrument. This shared resource was a major driver in the complexity of the encounter sequence design, as there were conflicting requirements for when navigation and science images needed to be taken. The final design for the imaging campaign was the culmination of years of work among the Scientists, Navigators, and Engineers.

\subsection{Autonomous Navigation}

Unique to Deep Impact's encounter was the use of fully Autonomous Navigation to coordinate final events around impact. The technical challenge of synchronizing the flyby spacecraft with impactor events well enough to image the impact and subsequent crater formation was a major design element for the overall comet encounter activity. This section introduces AutoNav as used for Deep Impact.

The autonomous navigation (AutoNav) system for Deep Impact was designed to control pointing of the instruments on-board the Flyby. AutoNav also provided sequence timing updates required to support high-rate imaging at the time-of-impact (TOI) as well as initiate the transition to shield mode at the correct time. The system had two primary objectives: 1) Capture the impact event in both the MRI and HRI cameras and 2) track the impact site for at least 800 seconds following impact to monitor ejecta plume dynamics, crater formation, and capture the highest possible resolution images of the fully developed crater before shield attitude entry. AutoNav relied on the performance of and interaction with the Attitude Determination and Control System (ADCS) flight software and subsystems as well as the MRI camera, which served as both a Science instrument and the primary AutoNav camera.

The AutoNav software was developed for the New Millineum Deep Space 1 (DS1) mission ${ }^{3,4}$. AutoNav consists of three (3) distinct modules: 1) Image processing; 2) Orbit determination; and 3) Maneuver computation. On the Flyby, only the first two modules were used during the final $2 \mathrm{hrs}$ of encounter with comet Tempel 1. AutoNav was originally developed to operate in two different modes: 1) Star-relative mode, which uses images that contain both the target body (beacon) and two or more stars for determining the orientation of the camera at the time of each image exposure; and 2) Starless mode, which uses the ADCS estimated s/c attitude and camera alignment information to determine the orientation of the camera at the time of each image exposure. For Deep Impact, the Starless AutoNav mode was used based on the expected quality of the ADCS estimated attitudes. The combination of the two CT-633 Star Trackers and SSIRU rate sensor provided an estimated attitude bias of no more that $150 \mu$ rad $(3 \sigma)$, bias stability of $50 \mu \mathrm{rad} / \mathrm{hr}(3 \sigma)$, and estimated attitude noise of $60 \mu \mathrm{rad}(3 \sigma)$.

The steps involved in the autonomous tracking process are as follows:

$\square \quad$ Acquire MRI images of the comet nucleus, every $15 \mathrm{sec}$, starting $2 \mathrm{hrs}$ before the expected time of impact

$\square \quad$ Process MRI images to compute pixel/line location of the nucleus center of brightness (CB) using the Blobber algorithm for the first $60 \mathrm{~min}$

Use observed $\mathrm{CB}$ pixel/line locations to compute measurement residuals for comet-relative trajectory estimation

口 Perform trajectory determination updates (OD), every $1 \mathrm{~min}$, starting $1 \mathrm{hr} 59 \mathrm{~min}$ before the expected TOI 
$\square \quad$ Produce and hold two (2) sequence time updates, with every OD update, to start the TOI imaging sequence and to control the timing of the final imaging sequence and entry into shield attitude for inner coma passage

口 Perform Scene Analysis (SA), 4 min before impact using the MRI, to point instruments at the impact site

口 Provide sequence time updates upon request (via command) from the critical sequence

․ Provide comet-relative velocity information to align solar array edge in the direction of on-coming dust flow

Image processing for the AutoNav system served the purpose of providing observations of the spacecraft cometrelative trajectory over time. When images were received by AutoNav, they could be processed in one of three ways: 1) Brightness centroiding of all pixels above a brightness threshold and within a predetermined pixel subregion (Centroid Box); 2) Image blobbing to detect one or more contiguous regions of pixel brightness above a brightness threshold and compute the pixel/line location of each blob based on a simple moment algorithm (Blobber); or 3) Scene analysis to compute the pixel/line offset, relative to the CB location as determined from either 1) or 2) above, of the region most suitable for an illuminated impact.

Brightness centroiding and image blobbing are well-known algorithms. Scene Analysis, however, was developed specifically for Deep Impact. Scene Analysis was developed by George Null and enhanced by Nickolaos Mastrodemos at the Jet Propulsion Laboratory. Mastrodemos discovered that Scene Analysis, while improving the number of illuminated impacts, could drive the impact site to a region that is obscured from view as the Flyby passes beneath the nucleus depending on the orientation, size and shape of the nucleus. Mastrodemos' version provided AutoNav with the ability to target a specific illuminated location on the nucleus in a biased fashion. The biasing drove the site selection to a location that was "biased" toward the Flyby's point of closest approach, thus enhancing the ability of the Flyby to image the fully developed crater at the time of highest resolution. Here, images were first processed using either the Centroid Box approach or the Blobber algorithm to provide a reference CB location. Scene analysis was applied and the pixel/line location of the selected site returned. The difference between the CB reference location and the selected site was computed and converted to an inertial correction vector that was stored and passed to ADCS for pointing control. If no suitable site was found, then no correction vector was returned which resulted in a default to tracking the CB.

For the Flyby s/c, the trajectory was estimated and updated every minute during the last $2 \mathrm{hrs}$ of encounter. The trajectory determination software supported both ADCS attitude control and sequence timing. The orbit determination (OD) arc length was selected to be 20 min for Deep Impact (based on flyby tracking performance as modeled over a range of nucleus models) and with image processing every $15 \mathrm{sec}$, each OD arc contained 80 observations. Following completion of each OD, the trajectory was updated and a representation of the estimated trajectory generated in the form of Chebyshev polynomial coefficients (Chebys). These Chebys represented a time series of the predicted Flyby positions relative to the nucleus and were passed to the ADCS flight software for evaluation and pointing control. ADCS evaluated the Chebys, computed the relative position vector, applied the SA offset and aligned the HRI camera boresights with the comet-relative position vector to center the nucleus in the instrument FOV.

\subsection{Robustness}

Several flight software features were used for encounter making it a robust, fault tolerant activity. The key fault protection design impact was in the sequence architecture. In addition, the enabling/disabling strategy employed for encounter was intended to improve the flight system's ability to press ahead with critical events without interruption as impact approached. The flight team also prepared several major encounter contingency plans, and designed the final encounter activity to succeed even in the event of a major fault.

As discussed in the sequence timing design sections, mark and rollback was a key feature used to improve the robustness of the entire design. Mark and Rollback is a feature of the FSW Command Manager CSC which allows for an absolute-timed critical sequence to be restarted automatically after a fault has occurred. Each command in a critical sequence is a mark point, making the selection of commands in a critical sequence very important. Once the next command in a sequence has been executed by Command Manager, the sequence cannot roll back to the previous command. Each command in a critical sequence can have a timeout window associated with it. This defines the extent of time over which a rollback can occur. Once this timeout window has expired, the sequence will roll forward and wait for the next command in the sequence. 
The critical sequence for encounter was an absolute-timed sequence. The only commands in the critical sequence (its mark points) were spawn commands to start to sub-sequences. These sub-sequences were relative-timed and only contained spawns to absolute- and relative-timed sequences containing the spacecraft and instrument commanding. The spawns in the critical sequence to the sub-sequences had timeout windows which expired at the next command in the critical sequence. Of primary importance in critical sequence design was the selection of the break points in the sub-sequences. These breaks, each a Mark Point in the critical sequence, were chosen to occur at the completion of a critical event, such as Separation, so that in the event of a fault, an important activity would be completed prior to rolling forward to the next task. In addition, some mark points were chosen to accommodate contingency plans, ie. swapping in alternate imaging sequences in the face of unexpected cometary environments.

The strategy for autonomous fault protection during encounter was challenging due to the time critical and unknown environment aspects of the event. At the start of the critical encounter phase, the set of FP enabled was similar to the cruise setting, with additional instrument, attitude control (point state), and telecom (HGA) recovery enabled. As impact time approached, more and more fault protection was disabled to lessen the chance of interference with encounter. At impact minus two hours, spacecraft system safing was disabled (recovery from safing would not have allowed recovery in time for successful encounter operations after this time), leaving system responses (major hardware swaps, basic hardware protections, etc.) enabled. At impact minus seven minutes all system responses were disabled. From that time until impact, fault protection was in place for only low level hardware protection, and processor reboots.

In addition to on-board autonomous fault protection, several contingency plans were prepared in advance of the encounter activity. All sequences to support these plans were loaded into on-board memory with the nominal encounter sequence upload. The major encounter contingency plans were as follows ${ }^{5}$ :

$\square \quad$ Unexpected Comet Albedo - If Tempel 1 was significantly brighter or more dim than predicted, alternate imaging sequences would be executed (exposure durations were dependant on comet albedo).

$\square \quad$ Late Release - If in preparation for separation the Impactor Spacecraft experienced anomaly, the encounter timeline would be adjusted to perform separation at E-10 hours instead of the nominal E-24 hours

$\square \quad$ Flight System Impact- If the Flyby and Impactor were unable to separate, this contingency plan would execute encounter with both craft still joined.

Each of the key robustness considerations: mark/rollback, fault protection enabling/disabling, and contingency plans played a major role in the design of encounter. Sequence timing, architecture, and command content/selection were all selected in light of these factors. The final design proved resistant to faults, capable of recovery in the face of contingency, and ability to roll forward and continue the critical mission throughout the punishing test program discussed in the next section.

\subsection{Comet EnCOUnTER TESTING}

Developing the Tempel 1 encounter activity presented a complex, multi-disciplinary set of challenges. Following the design phase of encounter, it was necessary to develop and execute a test program that would verify and validate the design. The encounter test program was developed to test, in a flight-like manner, all aspects of the encounter activity. This included nominal sequence performance testing, off-nominal faulted testing, stress testing, and operations simulation testing. The program spanned two years, and had elements that were executed on all test venues, including the software test benches, the flight system test bench, and the flight system itself.

This section gives an overview of the Deep Impact encounter test program, including objectives, program architecture, and performance predictions.

\subsection{Encounter Test Program Objectives}

Encounter testing, in line with the overall project Verification and Validation program, was focused on the following major 'readiness' objectives:

․ Ensure that normal encounter operation does not trigger Fault Protection

$\square \quad$ Ensure that the system is robust under all expected encounter operating conditions

$\square \quad$ Ensure that system responses are predictable, and the results are acceptable 
$\square \quad$ Ensure that the system operates in off-nominal (within-specification) encounter conditions

口 Push the boundary conditions of the system to investigate the ability to operate outside of specification, and understand when overstress occurs and how it is handled

\subsection{Encounter Test Program}

Deep Impact relied heavily on the test program to prove flight system and flight team readiness for encounter. The design of this event was so constrained that testing was started early in the activity development program to help guide design decisions. This is unique when compared to most test programs; typically testing is used only after design is completed. Each of the readiness tests used for Deep Impact was absolutely essential in developing the design, and later verifying and validating it in preparation for comet encounter.

Each test conducted in this program was categorized as a Mission Readiness Test (MRT). The set of MRTs was composed of several types of system-level tests, defined below:

․ Mission Scenario Test (MST): MSTs are tests using flight-like, MOS/GDS-generated sequences in tests targeted to verify and validate key mission scenarios (such as encounter) that will be executed in flight.

$\square \quad$ Faulted Mission Scenario Test (Faulted MST): Faulted MSTs are tests that inject specific faults into a nominal MST run, and let the system respond. The objective of these tests is to prove appropriate system response to known off-nominal conditions, and to validate system recovery in the presence of faults.

口 Robustness Test: Robustness tests were intended to vary key performance parameters (ie. dust model, center of mass, etc.) by $\pm 3 \sigma$ and $\pm 6 \sigma$ to validate system robustness, and successful comet encounter in the event of stressors outside of specified performance.

$\square \quad$ Operation Readiness Test (ORT): An Operational Readiness Test uses flight-like, MOS/GDS-generated sequences/tools/processes in tests targeted to exercise system performance while training the flight team. Encounter ORTs were conducted in the flight Mission Support Areas with full flight team participation, including Flyby and Impactor teams for the individual spacecraft.

- Incompressible Encounter Test: Incompressible encounter tests are a subset of the full test set. These tests are designated as mandatory. In the case of Deep Impact Encounter, there was not an opportunity to waive off the event and try again later. The incompressible encounter tests included several key MSTs, Faulted MSTs, Robustness Tests, and ORTs. All were successfully executed prior to encounter.

Table 2: Flyby Encounter Test Summary

\begin{tabular}{|ccccc||}
\hline TEST TYPE & NUMBER OF CASES & TOTAL \# EXECUTED & \# ON ITL & TOTAL HouRS \\
\hline \hline MSTs & 3 & 279 & 3 & 5160 \\
Faulted MSTs & 16 & 25 & 8 & 300 \\
Robustness Tests & 11 & 35 & 11 & 288 \\
ORTs & 7 & 7 & 5 & 400 \\
Totals & $\mathbf{3 7}$ & $\mathbf{3 4 6}$ & $\mathbf{2 7}$ & $\mathbf{6 1 4 8}$ \\
\hline
\end{tabular}

Sheer complexity of the encounter event, likened to hitting a bullet with another bullet, was one of the biggest challenges in accomplishing the test program. System performance was stretched in almost every subsystem: images were taken at maximum speed, NVM was filled to the maximum, ADCS and AutoNav processing was at its peak, and telemetry bandwidth was maximized. These factors, coupled with the unknowns of the Tempel 1 environment (albedo, dust environment, size of the nucleus, rotation rate, etc.), and precise timing synchronization between both craft, demanded correct settings for thousands of parameters. Any one of those parameters, if wrong, could have compromised the mission. Table 2 shows a summary for the numerous test cases run for Flyby encounter, and highlights the large numbers of hours spent testing the final design. 


\subsection{Performance Predictions}

Upon successful completion of the Encounter Incompressible Tests, the flight team expected good performance from the flight system. All subsystems, including ADCS and AutoNav performed well time and again during the final ITL tests. Encounter tests predicted a successful hit by the impactor, and successful imaging of that spot by the flyby. The complicated data flow was difficult to verify, as test bench hardware was not of adequate fidelity to fully emulate the flight system performance. In addition to test bench runs, high fidelity data flow models were used to estimate performance. By the end of the test program, the data flow model was considered a good indicator for overall system performance. This model, when all systems were modeled to run correctly (ie. impactor hits comet, all critical images in data flow, imaging directions correct with large image sizes, slews/maneuvers successful, etc.), showed the closest comparison to in-flight performance during encounter demonstrations. The predictions shown below are results from the final data flow modeled runs of Encounter Approach and Encounter. These predictions were presented as verification data at the Critical Events Readiness Review prior to encounter.

Encounter Approach was predicted to successfully capture and downlink prior to deletion 1769 navigation images, 31903 ISI files, and 2616 science images, including those taken during the mission critical mini calibrations. A few navigation images (4) and Impactor ISI files (11) were predicted to be deleted prior to downlink to preserve the timely start of the mini calibrations. Bandwidth usage expectations were favorable for the entire week of continuous flyby and Impactor operations, showing no dropped image packets or interruptions in telemetry. Science NVM usage was predicted to fit within the $309 \mathrm{MB}$ allocation, and the downlink queue never exceeded the 300 file maximum in the final modeled run. All maneuvers, commands, and activities were successful in this modeled run. Figure 8 shows the fully modeled encounter approach predicted data flow performance.

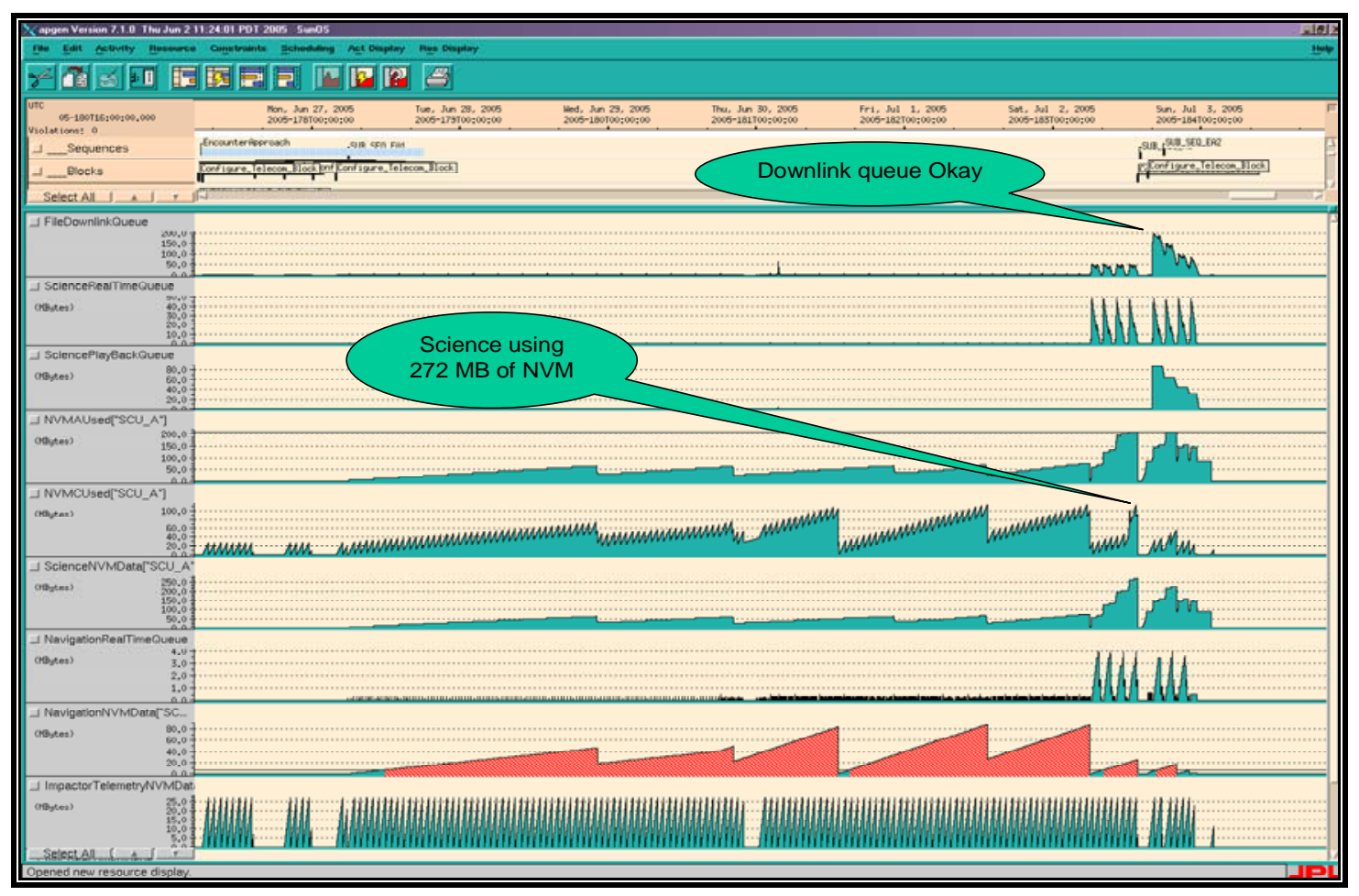

Figure 8: Encounter Approach Predicted Data Flow Performance

The critical encounter sequence was predicted to also perform flawlessly. Of the 6012 science, 199 optical navigation and 936 autonomous navigation images taken during the encounter sequence, predictions indicated all would successfully reach the ground prior to deletion. All Impactor images, including those closest to impact time ( $\sim 3$ seconds from impact) were also expected to reach the ground successfully. The "live for the moment" strategy to get all high priority images down prior to closest approach of the flyby to Tempel 1 was also predicted to be executed without incident. Science NVM usage was expected to fall below the 309 MB allocation, and the downlink queue was not predicted to exceed the 300 file maximum. During ORTs, executed on the test benches, there were 
significant delays and losses of Impactor telemetry during critical Impactor AutoNav operations and Impactor ITMs. Modeled runs also predicted some Impactor data loss, but modeling showed outages that represented less than three minutes of Impactor telemetry. The performance of the Command and Data Handling Subsystem (C\&DH) on the test benches was an issue throughout testing of the encounter sequences, causing loss of critical science images of impact and the crater formation. Images were essentially being taken faster than the C\&DH test bench hardware could process/store them. Modeled runs did not echo this poor performance in C\&DH-dropped images were seen only on the test benches. Final performance predictions were based on the modeled runs due to the differences in performance between the flight system and the test benches. All maneuvers, commands, and activities were successful in this modeled run. Figure 9 shows the final predicted performance for encounter data flow.

Final test executions showed excellent performance of all flight systems throughout encounter. TCM test runs were in line with earlier in-flight performance, and showed that TCM 5 should execute perfectly. ADCS and AutoNav were predicted to successfully execute their required functions, including delivering the Impactor onto an impact trajectory, and choosing an impact spot that the flyby could image at impact. The final runs of incompressible tests all resulted in Impactor "hits," even in the case of some off-nominal performance.

In-flight demonstrations were also an indicator of future performance at encounter. The Saturn Watch test, executed on the flight system during cruise, showed outstanding AutoNav performance in tracking Saturn and picking an impact location on that planet. The in-flight demonstration performance was as expected for the real Tempel 1 event. In addition, the in-flight encounter demonstration was quite successful. This demonstration executed the hardest part of the Flyby's sequence, including a demanding slew just after the impact images were captured, and demonstrated the end-to-end data flow from spacecraft to data archive. This demonstration included cooperation with the Deep Space Network to test the array of antennas schedule for encounter. The demonstration went very well-all test objectives were met. All test venues, from software simulations to the real spacecraft, showed predictions of a successful encounter with Tempel 1.

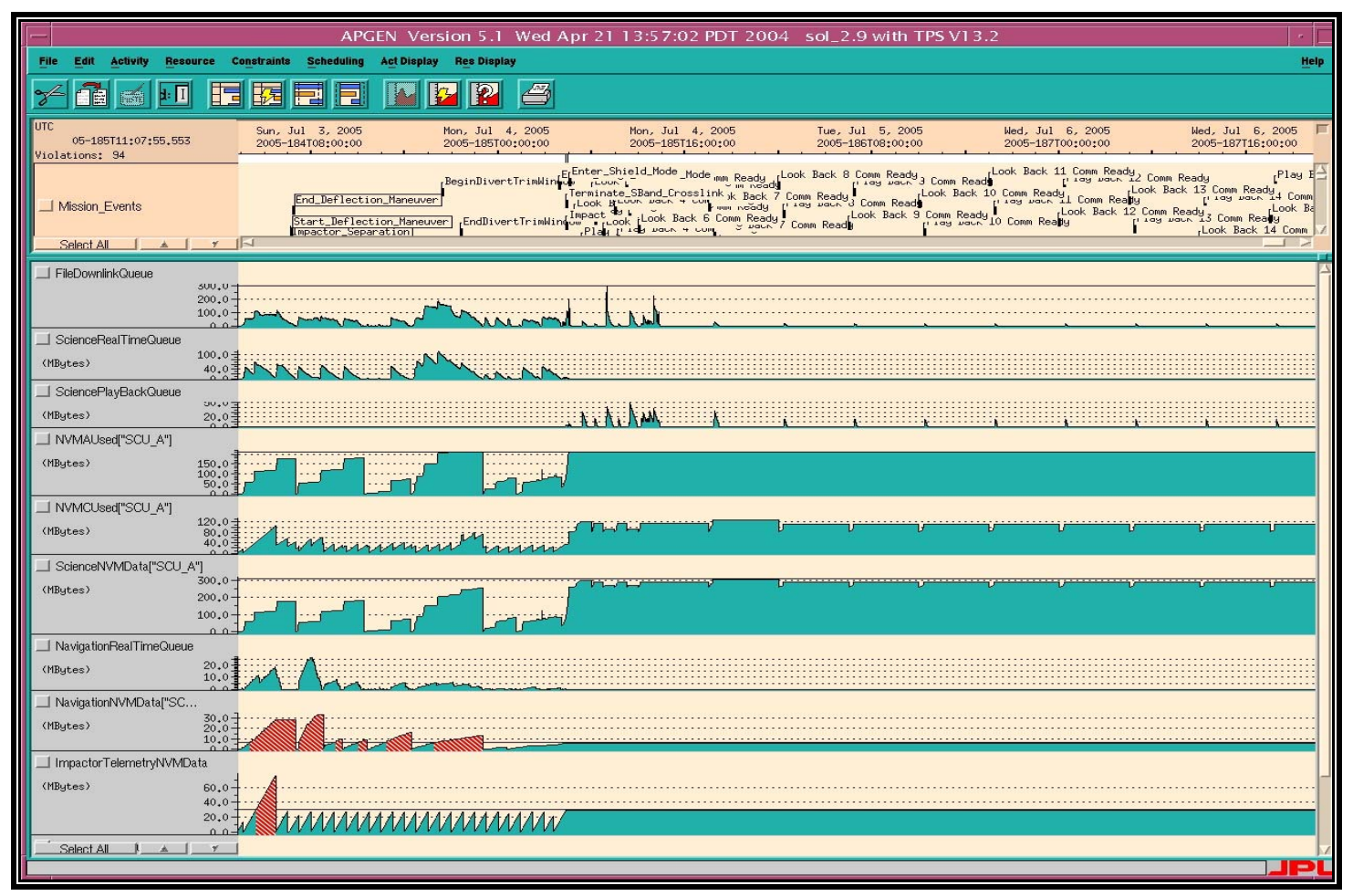

Figure 9: Flyby Encounter Predicted Data Flow Performance 


\subsection{Encounter Execution \& Performance}

The stunning images seen around the world on July 4, 2005 proved without a doubt that Deep Impact was a success. The following sections detail the flight system performance of the flyby spacecraft for this historic event.

\subsection{Encounter Approach Performance}

The week prior to encounter was filled with intense imaging, maneuvers, and calibrations. Figure 10 shows representative science images taken during the Encounter Approach phase. The images, from left to right, span 4 days of approach. The last image was taken July 1, the flight system was 2,446,529 km and less than three days out from Tempel 1.

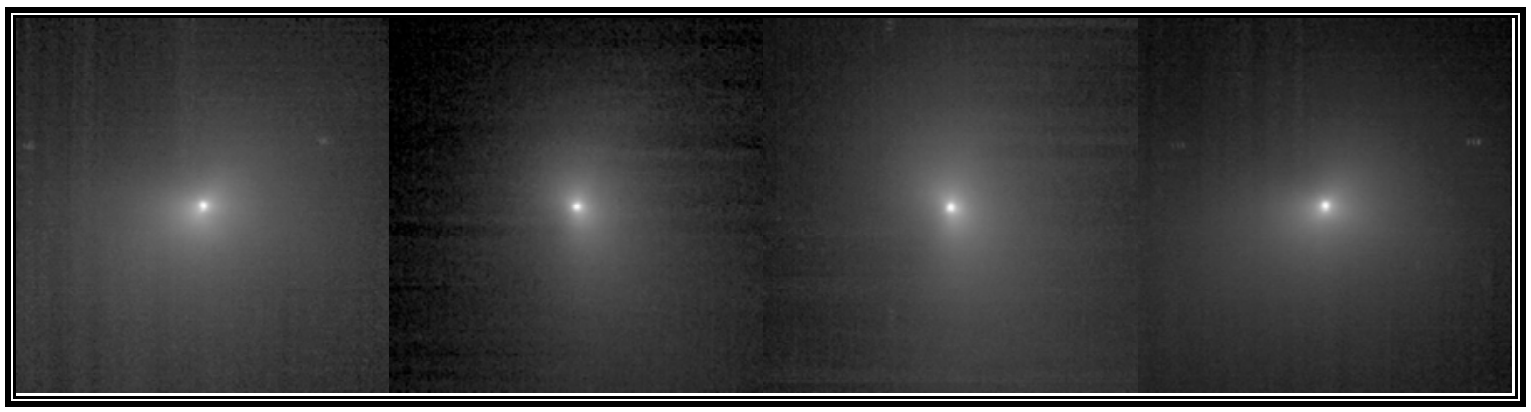

Figure 10: Encounter Approach MRI Clear Filter Images ${ }^{6}$

Of the 4389 science and navigation images taken during encounter approach, 127 did not reach the ground. All 127 images lost did not impact mission success. The lost images were deleted prior to downlink by the on-board sequence. It was found that a single over-subscription in real-time telemetry was the cause for the bandwidth overflow. This over-subscription fully accounts for the mismatch in performance to predictions.

The mini-calibrations performed for the science team to use for deconvolution of the HRI images could not have been more successful. The beautiful, high-resolution HRI images shown in the following sections are a direct result of the rapid turn-around from the Point Spread Function and Infrared Mini Calibrations.

All performance metrics for each subsystem, the flight team, and the science team, were met or exceeded for the final week prior to impact. Throughout the Deep Impact mission, Trajectory Correction Maneuvers were quite accurate. TCM 5 executed within $2 \%$ of desired performance. This placed the flight system with $1 \mathrm{~km}$ of an impact trajectory. The flight system encounter activity was designed to accommodate up to $30 \mathrm{~km}$ of Impactor delivery error, so the $1 \mathrm{~km}$ performance was outstanding. The final responsibility of encounter approach was to clean up NVM for the critical event. At the completion of the encounter approach sequence, all drives were clean saving only the files required to execute encounter.

\subsection{Separation/Divert}

The separation sequence executed nearly flawlessly. There was an excessive attitude error fault protection symptom raised at Impactor separation. Fortunately, fault responses were still disabled at this point, so no fault response ran. The error was due to a change in the timing of re-enabling attitude control after the separation event, and a lack of fidelity in testbed simulation to trigger the response during testing. The divert sequence also ran flawlessly. The divert performance resulted in approximately 1 second timing error in the time of impact, and $2 \mathrm{~km}$ error in closest approach distance. The flyby spacecraft successfully captured an image of the departing impactor, which appeared as a bright smear in the center of the FOV of the MRI.

\subsection{Autonomous Navigation}

The AutoNav system performed beyond expectations. Figure 11 is a compilation of images taken with both the MRI and HRI instruments at various times during AutoNav operations. 


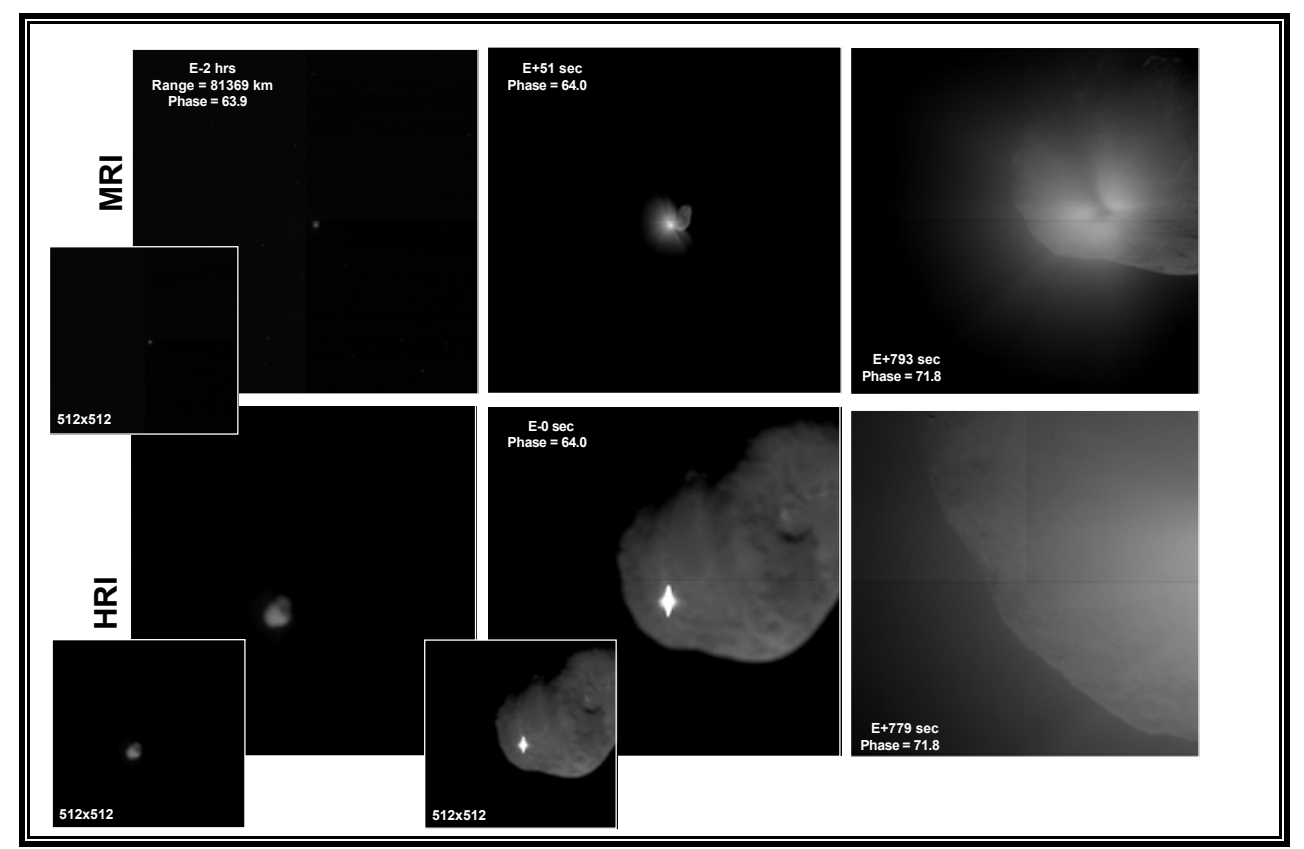

Figure 11: Set of MRI (top) and HRI (bottom) images taking during AutoNav operations ${ }^{6}$

All of the primary Flyby AutoNav objectives can be summarized in two images. Figure 12 shows the HRI image capture at the moment of impact. The saturated pixels are located within 31 pixels of the center of the HRI FOV. Each HRI pixel is 2 microradians and therefore indicates that the total of all error sources was less than 62 microradians at TOI. This includes errors associated with: 1) AutoNav estimation; 2) ADCS attitude estimation; 3) HRI camera alignment errors; 4) Independent Scene Analysis induced errors; and 5) Errors associated with updated estimates of TOI.

Figure 13 shows a full-frame MRI image of the impact site just $7 \mathrm{sec}$ before shield attitude entry. While the impact site was lost for the HRI FOV nearly $13 \mathrm{sec}$ earlier, the 3 picture mosaic (scan) that was implemented to increase the probability of capturing the crater in at least one of the last 3 images taken before shield mode, did just that and has given the Science Team an opportunity to quantify the size of the fully developed crater.

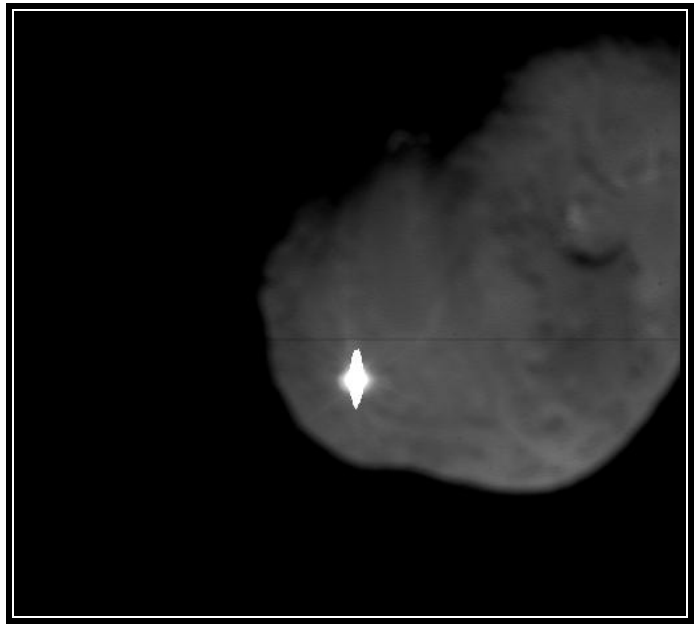

Figure 12: HRI 512x512 pixel subframe image showing initial flash at the moment of impact ${ }^{6}$

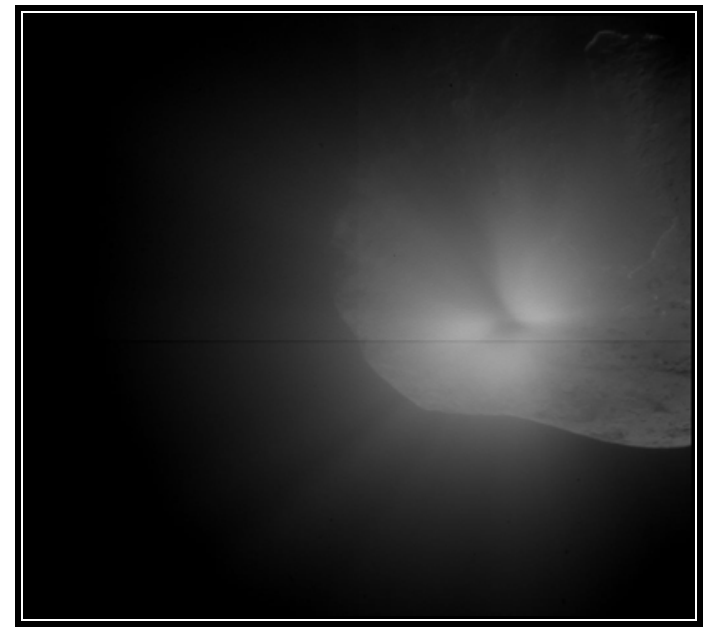

Figure 13: MRI Full-frame image of the impact site (center of ejecta cone) at 793 sec after impact ${ }^{6}$ 


\subsection{Encounter Imaging and Data Return}

The Flyby encounter Imaging campaign performed exceptionally well. Of the 6012 science images sequenced during the encounter phase, all of the pre-shield mode images taken after Impact minus 15 hours were received on the ground. Approximately 20 images from before Impact minus 15 hours were lost due to a failure in the DSN transmitter at one 70 meter ground station. The drop in gain from using a 34 meter station for uplink caused the loss of some transfer frames containing image file data. The downlink Signal-to-Noise Ratio (SNR) was improved by turning ranging modulation off at the 34 meter station. None of the critical impact or crater images were lost due to C\&DH performance. All but 66 of the over 6000 Impactor telemetry files were received without error on the ground and processed into Impactor telemetry displays. 63 (representing less than 15 minutes of Impactor telemetry) of these occurred early in the encounter period during the DSN transmitter failure. All critical Impactor telemetry was delivered to the Flight Team in a timely manner. Figure 14 shows encounter data flow performance, indicating the accurate prediction from the data flow model, as flight system actual performance matches quite well.

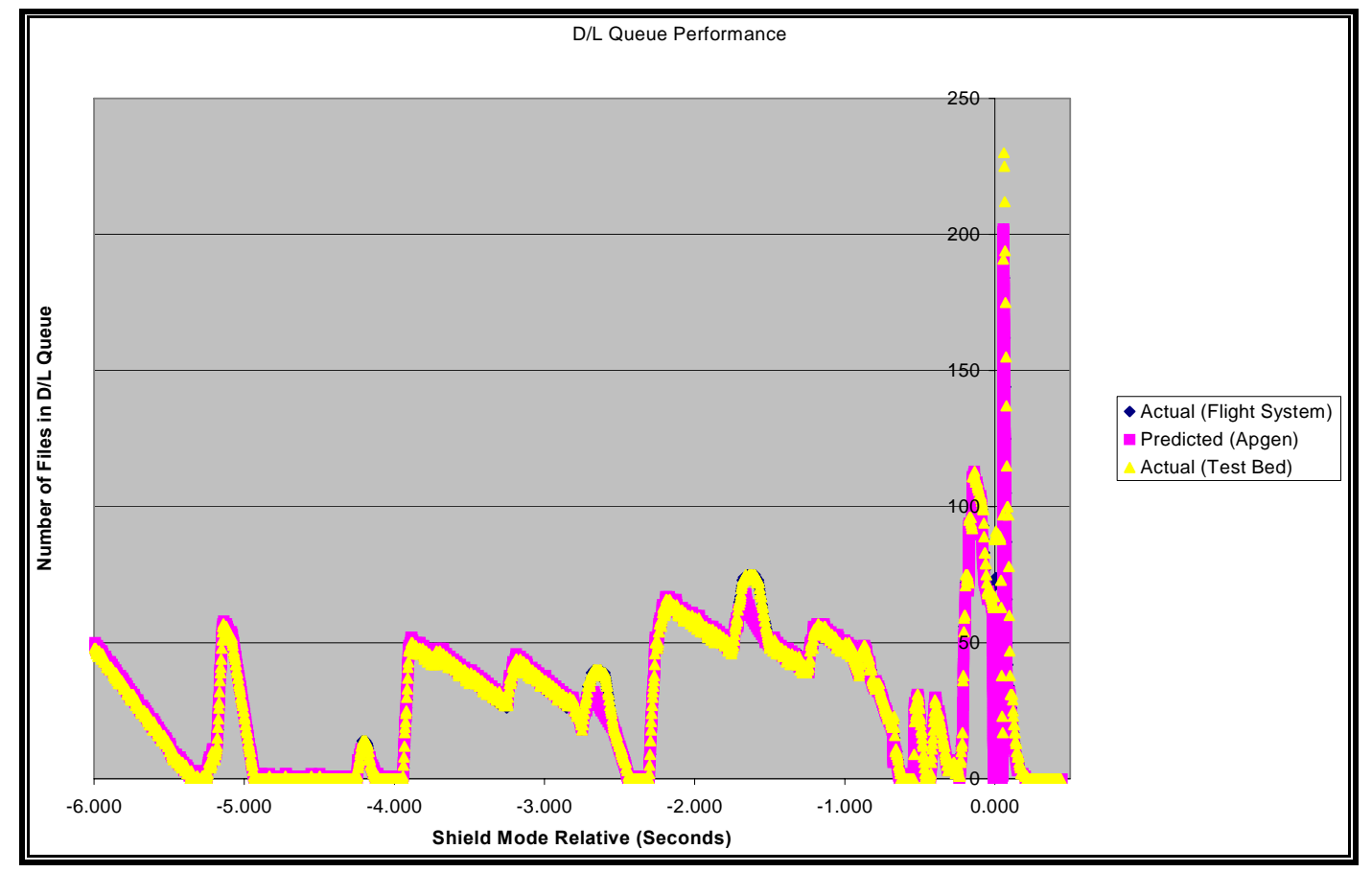

Figure 14: Encounter Data Flow Performance

Images downlinked from the Flyby during encounter were viewed in near real-time in the Mission Support Area. In addition to the engineering telemetry being tracked by each member of the flight team, the pictures of Tempel 1 just kept getting better and better. The well-scripted encounter event was captured in stunning detail- these images show the undeniable success of Deep Impact's date with comet Tempel 1.

This spectacular image of comet Tempel 1 (Figure 15) was taken 67 seconds after it obliterated Deep Impact's Impactor spacecraft. Scattered light from the collision saturated the camera's detector, creating the bright splash seen here. Linear spokes of light radiate away from the impact site, while reflected sunlight illuminates most of the comet surface. The image reveals topographic features, including ridges, scalloped edges and possibly impact craters formed long ago. 


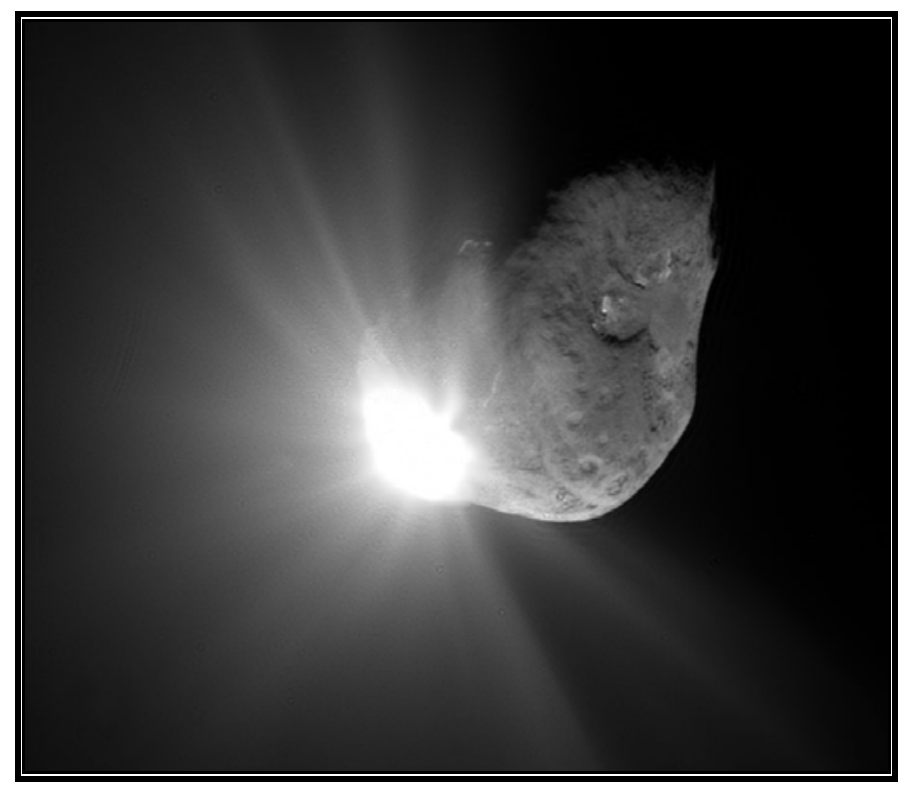

Figure 15: HRI Image of Tempel 1, 67 Seconds After Impact ${ }^{6}$

Figure 16 shows comet Tempel 1 about 50 minutes after Deep Impact's probe smashed into its surface. The impact site is located on the far side of the comet in this view. The colors represent brightness, with white indicating the brightest materials and black showing the faintest materials. This brightness is a measure of reflected sunlight. Because the sunlit portion of the comet is brighter, it appears white. The comet's nucleus is silhouetted against the light reflected from surrounding dust. The large plume of dust that was kicked up upon impact can be seen as the colorful, drop-shaped object. This plume was very bright, indicating that the comet's surface material must be very fine, like talcum powder. The blue speck in the upper left corner is a star.

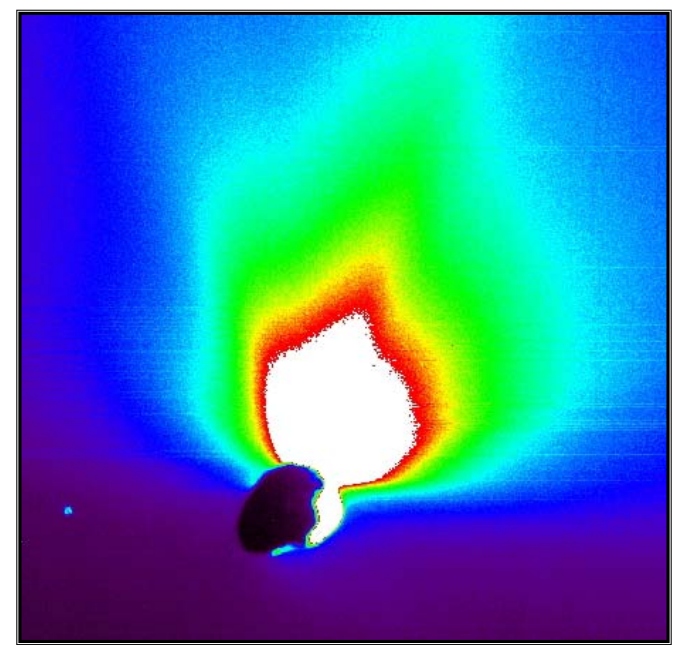

Figure 16: HRI Image of Tempel 1, False Color, 50 Minutes After Impact ${ }^{6}$

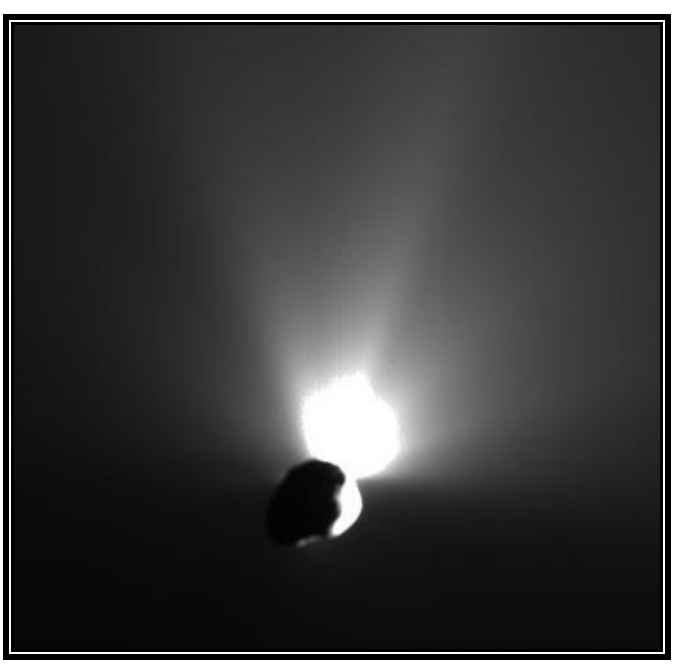

Figure 17: HRI Image of Tempel 1, 50 Minutes After Impact $^{6}$

Figure 17 shows the view from Deep Impact's flyby spacecraft as it turned back to look at comet Tempel 1. Fifty minutes earlier, the spacecraft's probe had been run over by the comet. That collision kicked up plumes of ejected material, seen here streaming away from the back side of the comet. $^{7}$

\subsection{Timing}

One of the biggest concerns about the unknown trajectory of Tempel 1 was the uncertainty in the time of impact and its effect on sequence timing. The original uncertainty estimate at the time the sequences were generated was 3 minutes. The timing updates generated by AutoNav for updates to the impact and high-resolution crater imaging 
sequences were on the order of 1 second on the day of encounter. The Navigation Team did an exceptional job determining Tempel 1's trajectory early enough to avoid large timing uncertainties at encounter.

\subsection{Tempel 1 Environment}

Throughout design, development, and flight, the question environment at Tempel 1 remained a mystery. In the final days leading up to encounter, Tempel 1 was active, with several unexpected "outburst" events that could have had implications on attitude control and AutoNav performance at encounter. The major elements of environmental concern were the dust environment and comet brightness (albedo). The flyby was designed to accommodate the dust particles up to $2 \mathrm{~mm}$ in diameter. Despite concerns about potentially damaging larger dust particles, on the day of encounter, the flyby spacecraft navigated the dust field without mishap. No major damage was sustained, and the flyby craft went on to produce the full set of planned look back images. One of the major contingency plans developed for encounter included alternate sets of imaging sequences in the event that Tempel 1 was significantly brighter or more dim than anticipated. For the Navigation team, the brightness of the comet had an impact AutoNav's ability to correctly choose the impact location. For the scientists, image exposure durations were highly dependent on comet albedo; appropriate exposure durations were required for mission success. On July 3-4, 2005, comet Tempel 1 had albedo in the nominal range expected for encounter. The flyby and Impactor spacecraft executed the baseline imaging sequences, yielding valuable images for the science team and nominal conditions for navigation.

\subsection{SUMMARY}

Deep Impact was a challenging mission. The major design elements of the encounter activity presented a difficult set of obstacles for the development team to overcome. The flyby spacecraft final encounter design was robust, met all constraints, and tested nearly every subsystem to its limit. A rigorous test program was employed to verify and validate the system design and performance. At its completion, the test program showed predictions of successful performance for the encounter. The engineering results from Deep Impact could not be more definitive. Stunning images of the impactor spacecraft obliteration and subsequent crater formation on Tempel 1 were viewed by astronomers across the globe and by space-based observatories. Despite engineering challenges, an unknown and potentially dangerous comet environment, and a short operations phase, Deep Impact was a dramatic success. This Discovery mission spent Independence Day with the world watching — what a sight!

The work described in this paper was carried out at the Jet Propulsion Laboratory, California Institute of Technology, under contract with the National Aeronautics and Space Administration.

\subsection{REFERENCES}

${ }^{1}$ Blume, W.H, and Wang, K.C, “Deep Impact Project Mission Plan,” 2004.

${ }^{2}$ Wissler, S, “Deep Impact Flyby Spacecraft Encounter Design,” 2005.

${ }^{3}$ Bhaskaran, S, Riedel, J. E, Synnott, S. P, “Autonomous Optical Navigation for Interplanetary Missions," Space Sciencecraft Control and Tracking in the New Millennium, Proc. SPIE, pp. 32-34, 1996.

${ }^{4}$ Bhaskaran, S, et al, "Orbit Determination Performance Evaluation of the Deep Space 1 Autonomous Navigation Software," AAS/AIAA Space Flight Mechanics Meeting, Monterey, CA, 1998.

${ }^{5}$ Sierchio, M, et al, "Deep Impact Encounter Contingency Plan Notebook,” 2005.

${ }^{6}$ Image Credit: NASA/JPL-Caltech/UMD

${ }^{7}$ Deep Impact Web Site, http://deepimpact.jpl.nasa.gov 